\title{
A Dynamic User-Centric Mobile Context Model
}

\author{
by

\section{Yu-Ling Chang} \\ A thesis \\ presented to the University of Waterloo \\ in fulfillment of the \\ thesis requirement for the degree of \\ Master of Mathematics \\ in \\ Computer Science
}

Waterloo, Ontario, Canada, 2010

C)Yu-Ling Chang 2010 


\section{AUTHOR'S DECLARATION}

I hereby declare that I am the sole author of this thesis. This is a true copy of the thesis, including any required final revisions, as accepted by my examiners.

I understand that my thesis may be made electronically available to the public. 


\begin{abstract}
Context-aware systems can dynamically adapt to user situations to provide smarter services. In general, context refers to the information that can be used to characterize these situations, and context models are deployed to specify contextual information described in context-aware systems. However, even though user context is highly dynamic, existing context models either focus on modeling static views of context or lack appropriate design abstractions to deal with dynamic aspects and interactions involving contextual elements such location, time, user roles, social relationships, and changing preferences. Moreover, virtual environments have not been modelled by most of the existing context models even though online interaction is very common and popular. This thesis presents a dynamic user-centric context model that can be used to model the aspects of context-aware systems that are subject to frequent change. Four case studies are proposed to illustrate the applicability of the approach taken by this thesis, and they are in the domains of mobile e-healthcare, mobile commerce, mobile tourism, and mobile augmented reality gaming. Benefits of the proposed model include avoiding the development of context-aware systems from scratch, enabling future use of modeldriven approaches, and reducing implementation effort.
\end{abstract}




\section{Acknowledgements}

I first would like to thank Professor Paulo Alencar for his excellent supervision. His positive encouragement has guided me to complete this work, and I am very grateful for his help and support. I would also like to thank Professor Daniel Berry and my thesis readers, Professor Donald Cowan and Professor Ladan Tahvildari, for their helpful comments. I would like to thank Eduardo Barrenechea who has provided tremendous help and insights into the work. I also would like to thank Dr. Rolando M. Blanco for his input in all the discussions and meetings. Kah-Kuen Fu has provided incredible support and helpful advice in many aspects of my graduate study, and I thank him for his wonderful friendship. This work would not have been possible without all the help from my thesis committee and research group.

I would like to thank my parents, Attlee and Sophia, for their guidance and encouragement. They are always positive and encouraging in every circumstance. They have motivated my dream in assisting people's work and lives through computers. I am deeply grateful for their love and support. I also thank my two siblings, Grace and Howard, for their support.

I would like to thank my housemates, Claire and Fanny, for all the late night chats and the amazing lunchboxes. The members of MCCF have provided wonderful friendships, and I truly appreciate their valuable emotional support for me. I especially would like to express my gratitude to Annie Lee who has been a great mentor and a role model of me. Francis Chen has provided great insights and help in many aspects of graduate studies. I also thank Angela, Jeff, Melo and Gary for their effort in making my office a less lonely space.

Finally, I thank God for His grace and all these wonderful people He has given to me. I especially thank God for David Lee whose patience and encouragement have supported me through all the difficulties and obstacles. His love, prayer and faith have greatly encouraged my journey with God in whom I found peace, joy and strength. 


\section{Dedication}

This thesis is dedicated to God for He has enabled me to complete this work. 


\section{Table of Contents}

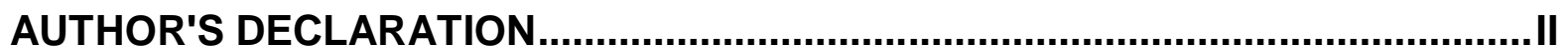

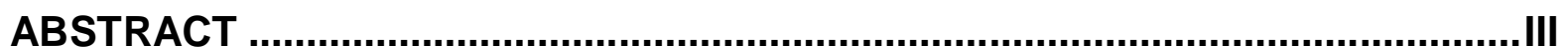

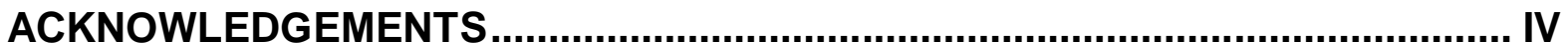

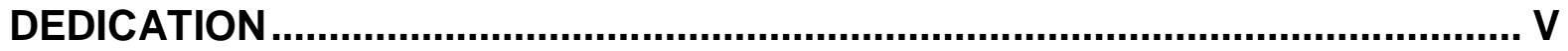

TABLE OF CONTENTS

LIST OF FIGURES

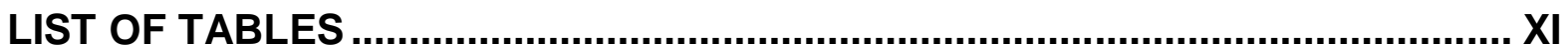

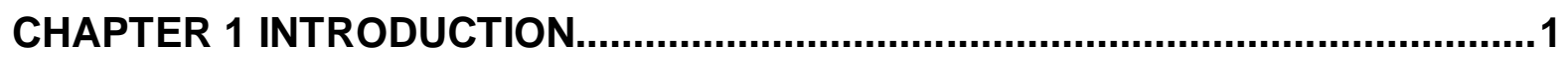

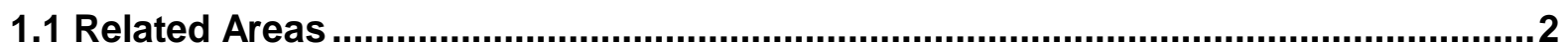

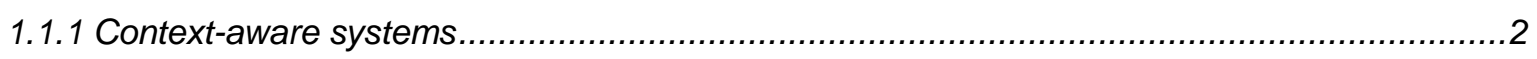

1.1.2 Design models and context models ...........................................................

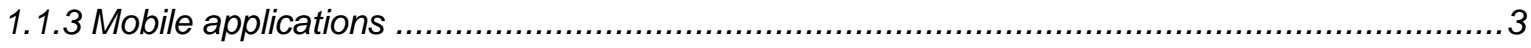

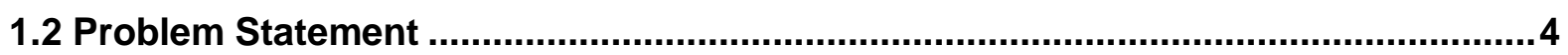

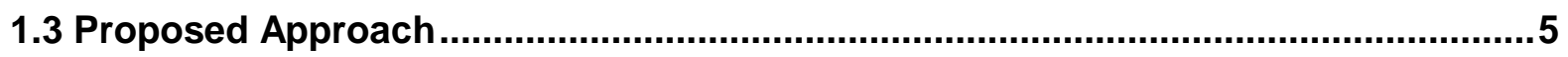

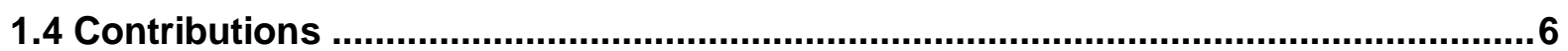

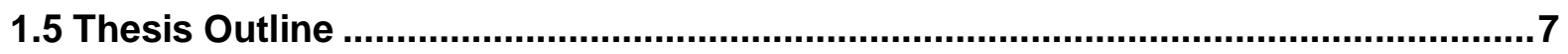

CHAPTER 2 RELATED WORK

2.1 Mobile Contextual Elements and Mobile Context Models ...........................................

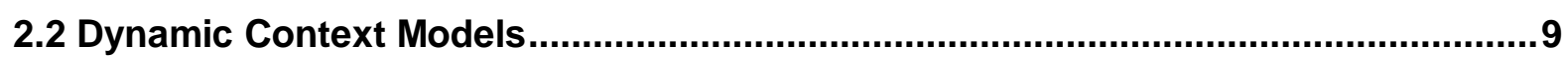

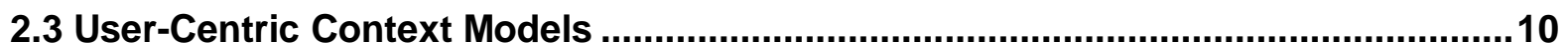

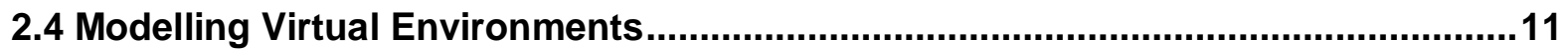




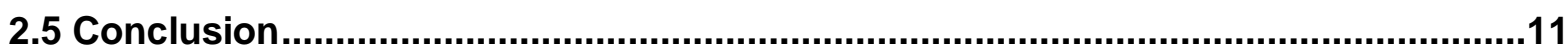

CHAPTER 3 CONTEXT-AWARE SYSTEMS ...................................................... 12

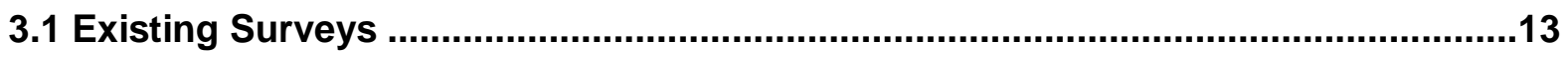

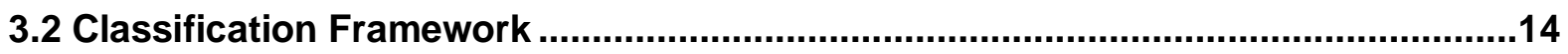

3.2.1 Fundamental element classification framework ....................................................... 15

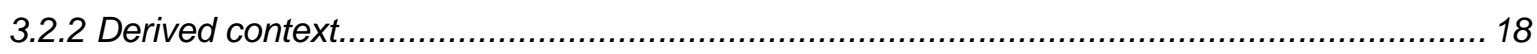

3.2.3 Functionality classification framework ......................................................................... 19

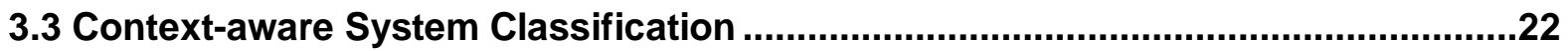

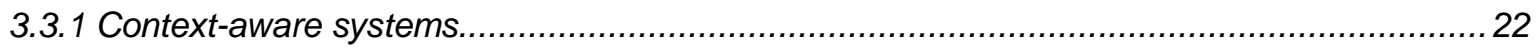

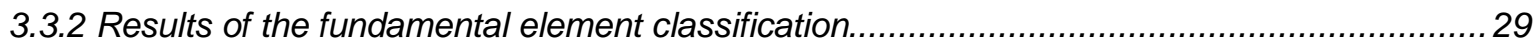

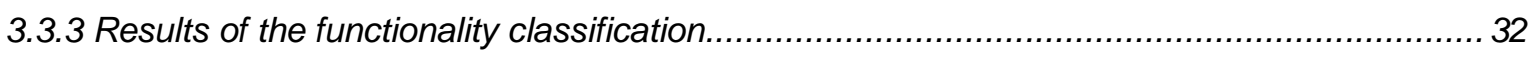

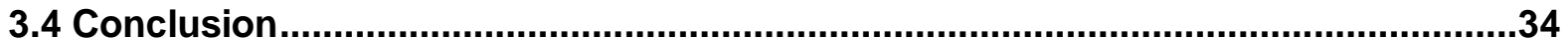

CHAPTER 4 A DYNAMIC USER-CENTRIC MOBILE CONTEXT MODEL ............. 36

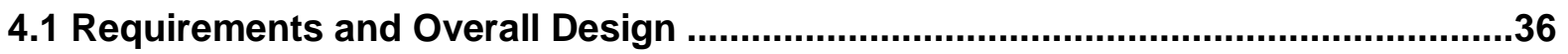

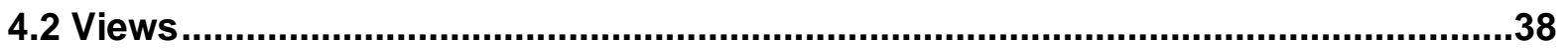

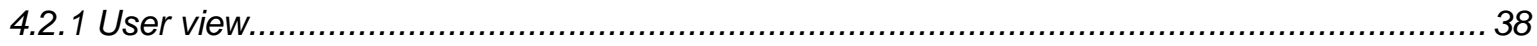

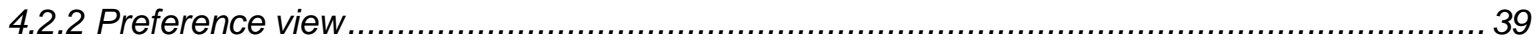

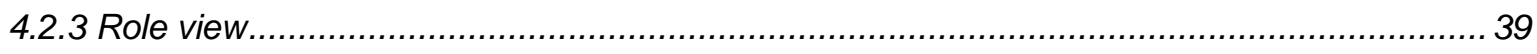

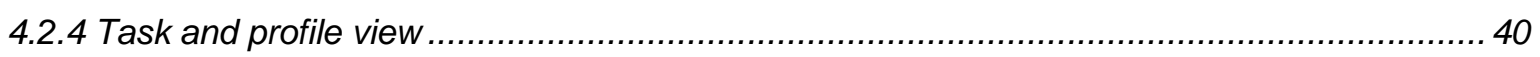

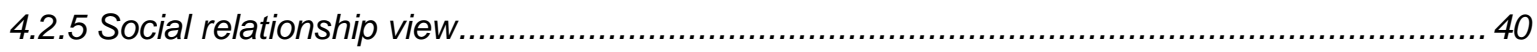

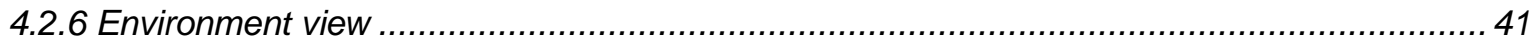

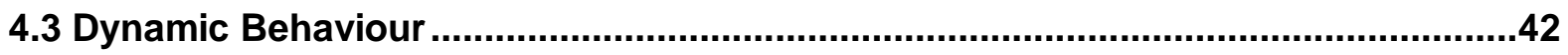

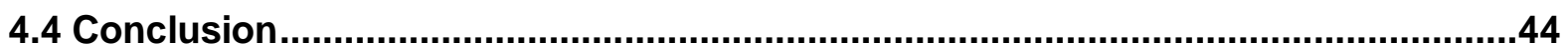

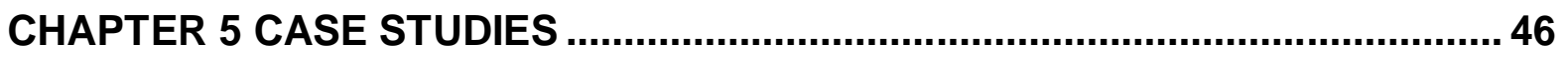

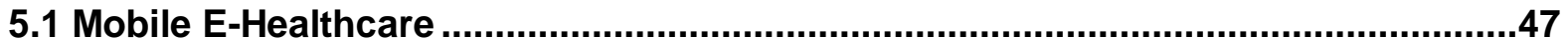




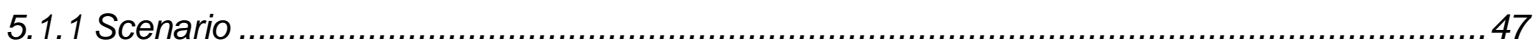

5.1.2 Dynamic interactions between contextual elements..................................................... 48

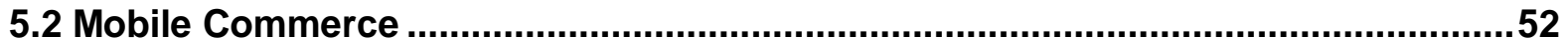

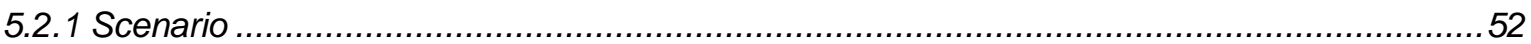

5.2.2 Dynamic interactions between contextual elements .................................................... 54

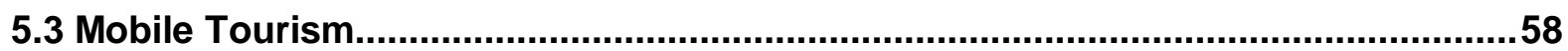

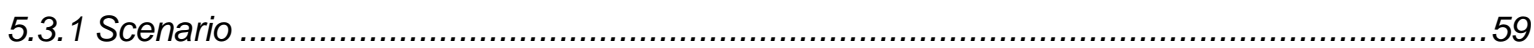

5.3.2 Dynamic interactions between contextual elements....................................................6 60

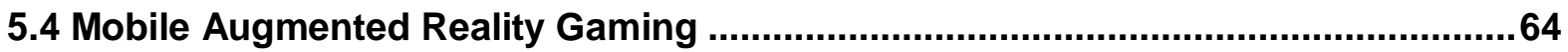

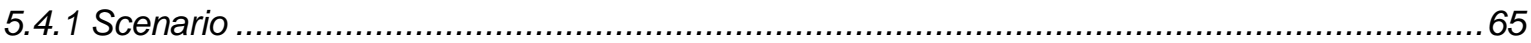

5.4.2 Dynamic interactions between contextual elements ................................................6 66

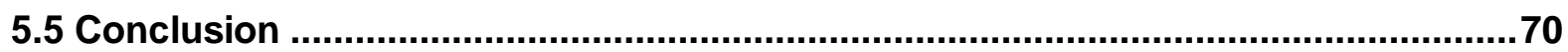

CHAPTER 6 CONCLUSION AND FUTURE WORK ….........................................72

6.1 Conclusion ................................................................................................................

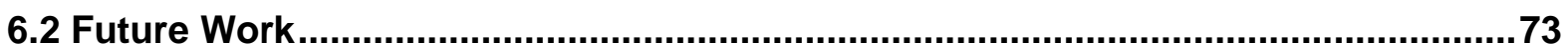

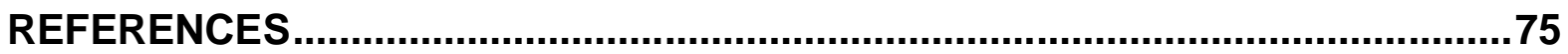




\section{List of Figures}

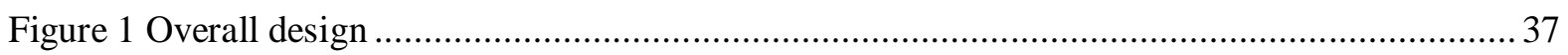

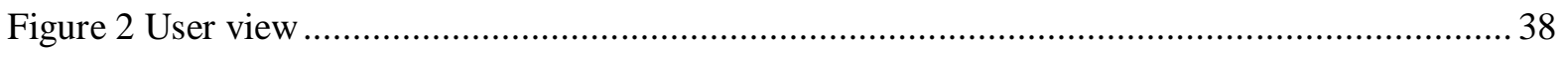

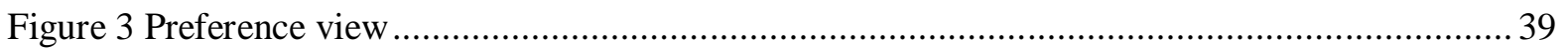

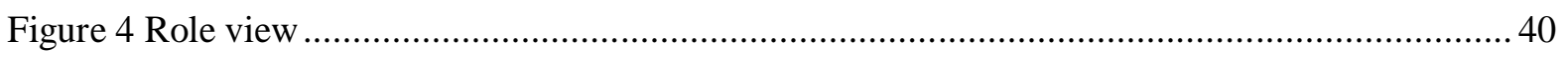

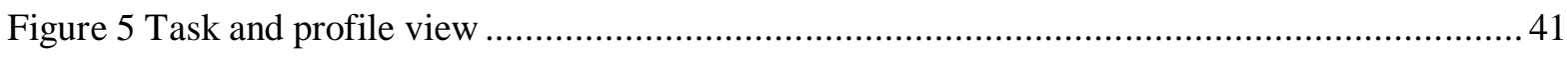

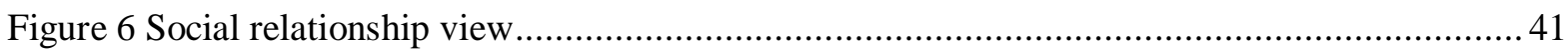

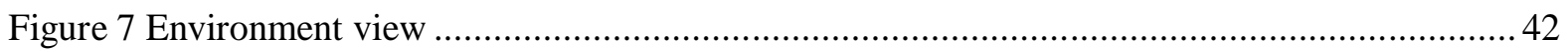

Figure 8 Dynamic interactions between task, profile, role and preference context elements .............. 43

Figure 9 Dynamic interactions between task, role and preference context elements ........................ 44

Figure 10 Object diagram describing changes triggered by environments changes in the mobile e-

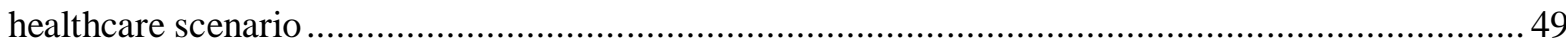

Figure 11 Sequence diagram describing changes triggered by environment changes in the mobile e-

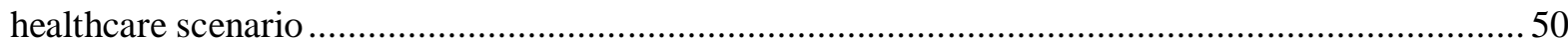

Figure 12 Object diagram describing changes triggered by task change in the mobile e-healthcare scenario

Figure 13 Sequence diagram describing changes triggered by task change in the mobile e-healthcare scenario

Figure 14 Object diagram describing preference adaptations triggered by role change in the mobile commerce scenario

Figure 15 Sequence diagram describing preference adaptation triggered by role change in the mobile commerce scenario

Figure 16 Object diagram describing preference adaptations triggered by role and task changes in the mobile commerce scenario

Figure 17 Sequence diagram describing preference adaptations triggered by role and task changes in the mobile commerce scenario

Figure 18 Object diagram describing the dynamic interactions between preferences and groups in the mobile commerce scenario .58

Figure 19 Sequence diagram describing dynamic interactions between preferences and groups in the mobile commerce scenario 
Figure 20 Object diagram describing the impact of work-related social relationships on the preference context in the mobile tourism scenario

Figure 21 Sequence diagram describing interaction between work-related group and preferences in the mobile tourism scenario

Figure 22 Object diagram describing the impact of tour partner and tour group members on the

preference context in the mobile tourism scenario.

Figure 23 Sequence diagram describing interactions between tour partner, tour group members and preferences in the mobile tourism scenario.

Figure 24 Object diagram describing the dynamic interactions between virtual environments, roles and preferences in the mobile augmented reality gaming scenario.....

Figure 25 Sequence diagram describing dynamic interactions between virtual environments, roles and preferences in the mobile augmented reality gaming scenario .68 Figure 26 Object diagram describing the dynamic interactions between tasks, groups and preferences in the virtual gaming environment in the mobile augmented reality gaming scenario. .69 Figure 27 Sequence diagram describing dynamic interactions between tasks, groups and preferences in the virtual gaming environment in the mobile augmented reality gaming scenario. 


\section{List of Tables}

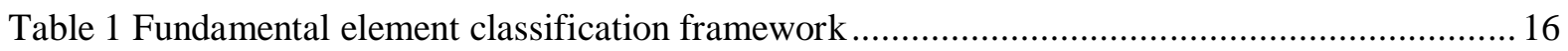

Table 2 Functionality classification framework......................................................................... 20

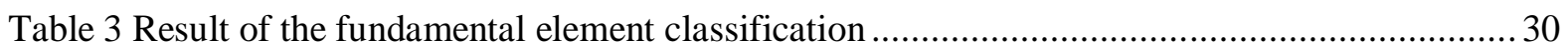

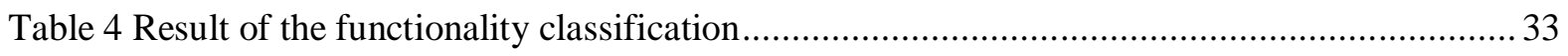

Table 5 Summary of dynamic interactions shown by each case study ....................................... 47 



\section{Chapter 1}

\section{Introduction}

Context-aware systems can dynamically adapt to specific user situations to provide smarter services and reduce the frequency of required manual inputs. In general, context refers to any information that is relevant to the user and his/her surroundings. Context is defined by Abowd et al. [1]:

"Context is any information that can be used to characterize the situation of an entity. An entity is a person, place, or object that is considered relevant to the interaction between a user and an application, including the user and applications themselves."

Context-aware systems are powerful and convenient because they are able to gather information and act based on it with minimal user interaction. They are used in various domains such as a smart home [2] or office [3], museum guidance systems [4], and Web services [5]. However, context-aware systems also pose interesting research questions. For example, context needs to be represented uniformly to allow communications among different systems. The systems need to provide timely services while processing large amount of raw and sensor information. As well, the input data received may be incomplete or inconsistent, but the systems still need to behave consistently and robustly. For the users of context-aware systems, there is a need to preserve their privacy and the security of their data since various types of information are gathered about them. Lastly, there is a need to abstract the capabilities of context-aware systems by developing reusable components that reduce the implementation effort and incorporate context at higher level of abstractions.

This research focuses on the context models deployed by context-aware systems. The benefits of having context models include being able to abstract and represent relevant contextual information used in context-aware systems. Focusing on context models also avoids the development of contextaware systems from scratch each time a new system is needed. As well, this approach enables future use of model-driven approaches and reduces implementation effort. By improving the expressiveness 
and completeness of context models, context-aware systems are able to provide more adequate and user-friendly services to users.

A context-aware system's capability to provide highly personalized and timely services has motivated its applications in the mobile domain. Mobile devices are usually owned by one user, and unlike computers they are not shared among family members. Because mobile devices are usually carried by users, a personalized mobile device can greatly benefit its user by providing comprehensive and adequate assistance based on its current situation. However, current mobile devices allow only a few degrees of personalization, such as changing themes and installing new applications. Context-aware mobile applications will be able to provide timely, personalized and suitable services to users and reduce the manual tasks. This thesis focuses on a dynamic and usercentric context model that is applied to the mobile application domain.

The rest of this chapter introduces the related areas, the problem, the approach, and the contributions of this thesis.

\subsection{Related Areas}

This thesis presents a context model, and the areas that are related to the model include context-aware systems which deploy context models, design models, and mobile applications.

\subsubsection{Context-aware systems}

Context-aware systems have been proposed in various domains ranging from application-level implementations to frameworks and context models. For example, the UbiqMuseum project [6] makes use of the Bluetooth technology to provide museum exhibit visitors with information that is suitable for their knowledge level and their language preference. For shoppers, SMMART [7] provides personalized promotions for customers close to or inside a retail store based on their shopping habits and preferences. CAPSIS [8] is designed to improve patient safety in the operating room during surgery by applying context-awareness to safety-critical systems. LoCa [9] provides a context-aware infrastructure that has dynamically adaptable user interface and workflow, and it can be used to monitor patients who are out-of-hospital in a digital home environment. Context Toolkit $[10,11]$ hides the complexity, abstracts context information, and provides building blocks to aid the development of context-aware applications. With a similar purpose, Vuković, Kotsovinos and 
Robinson [12] propose a framework to compose context-aware systems rapidly based on user requests and current demands.

\subsubsection{Design models and context models}

Blaha and Rumbaugh define a model to be "an abstraction of something for the purpose of understanding it before building it" [13]. It reduces the complexity, gives an abstract view, and helps in understanding the problem. A model can be reused to reduce the implementation effort. This thesis proposes a context model design. Context model captures the relevant contextual information to be considered in context-aware systems, and it provides a way to structure the contextual information. A contextual element needs to be considered in the model for the context-aware system to support related functions. The contextual information can be categorized into different dimensions, such as social relationships, user roles, location, time, and environments. These dimensions can be seen as the fundamental primitive elements in a context model.

\subsubsection{Mobile applications}

A mobile application is the software application running on a handheld mobile device, and it may provide basic functionality, such as calling and texting, or more advance functionality, such as gaming, streaming video and browsing the Web. The case studies in this thesis are about some types of mobile applications including mobile e-healthcare, mobile commerce, mobile tourism, and mobile augmented reality gaming.

The benefits of deploying computer-based systems and digitizing data have motivated the health industry to develop health systems that replace the traditional paper-based approach. For healthcare professionals, e-healthcare systems allow them to access the patient records electronically, and mobile e-healthcare systems provide this accessibility anytime and anywhere.

Mobile commerce enables business related activities such as transactions and advertisements to be performed through mobile devices with Internet connectivity. For example, a user may purchase items or receive promotions through their mobile devices. It provides customers or subscribers with anytime-anywhere shopping experiences.

Mobile tourism enables richer and more personalized tour experiences by providing extra information and services related to attraction sites through mobile devices. For example, mobile 
devices may provide navigation help, information on the historical background of an attraction site, and recommendations for dining.

Augmented reality connects the physical and the virtual world by superimposing virtual objects on top of the real environment. A user sees that the virtual and the real objects coexisting at the same time [14]. The advances in hardware enable the deployment of augmented reality applications on mobile devices [15], and this approach has made augmented reality applications affordable and easily accessible. A mobile augmented reality game provides players a different flavour of gaming world in which the physical and the virtual environments are seamlessly connected through mobile devices. Moreover, the mobility aspect provides an extra dimension to the game. Players can enjoy both the mobility and the fantasy at the same time, and they experience both the physical and the virtual environments together as well.

Some mobile applications are context-aware. They range over various domains such as language learning [16, 17], social recommender systems [18], and tour guiding [19-21]. Location context has been widely used by mobile applications [22, 23], and other contextual elements are starting to become popular to provide more personalized functionality. For example, CAESAR [18] makes use of social networks. The context-aware browser [24] makes use of user preferences, location, and activity. Ogata and Yano [17] propose to make use of role context to help foreigners learn Japanese polite expressions. The shift from location awareness to context awareness provides users with more powerful user-centric context-aware mobile applications that assist users' lives and work more adequately.

\subsection{Problem Statement}

The problems that have motivated the proposed design can be summarized as the need of context models that consider dynamic context interactions, user contexts, virtual environments and mobility.

Context is highly dynamic and is constantly subject to change. A change in one contextual element may propagate to other contextual elements. These changes are the dynamic interactions between contextual elements. For example, after a user arrives at work during work hours, the role of the user changes. In addition, the user may have a different set of preferences related to the new role. In order for context-aware systems to adapt to highly dynamic user situations, context models need to capture the dynamic interactions between contextual elements. However, the dynamic interactions between 
contextual elements have not been fully represented by the existing context models which often focus on modeling static associations among different contextual elements. In the existing context models, there is a lack of appropriate design abstractions to deal with dynamic aspects and interactions involving contextual elements such as location, time, user roles, preferences, and social relationships.

Context-aware systems need to assist users and provide user-friendly and suitable services by adapting to users' current situations. However, most of the current context models focus on contextual elements related to underlying devices and network implementation infrastructures. Users need to be the main focus to provide appropriate help. The existing context models lack abstractions to model user context, especially in roles, preferences, and social relationships.

The advancement and prevalence of the Internet and personal computing and communication devices have made online interaction very popular and easily accessible. People can access the Internet anywhere and anytime through various devices such as computers and mobile devices. We can also collaborate over great distances through the Internet using means such as video conferencing. A user may be situated in the physical and the virtual environments at the same time. However, current context models focus more on physical environments and locations. There is a lack of abstractions that model virtual environments.

Applying context-awareness to mobile applications will benefit users by providing them with personalized and timely services suitable to their current situations. However, current context models have not been widely applied to the mobile application domain.

\subsection{Proposed Approach}

This thesis proposes a general context model instead of a domain specific model. The four case studies instantiate the general model to specific mobile application domains, and they show the applicability and different aspects of the model. These case studies also illustrate that the context model is able to support the new trend of context-aware systems.

The context model reflects the dynamic interactions among contextual elements by modeling the association between them. Dynamic associations such as influence and enable are used to capture the changes triggered by changes in other contextual elements. 
This thesis focuses on a user-centric context model. The context model considers the dynamic interactions among fundamental contextual elements as well as three essential user context elements which are user roles, preferences, and social relationships. Each user has a set of roles and preferences. One of the roles would be the dominant role at one point in time, and this role would take precedence over other roles. One set of the preferences would be the dominant set at one point in time. For social relationships, the context model considers the users involved in the relationship as well as related contextual information such as common interest and location. Context-aware systems will be able to provide more adequate recommendations and services by considering extra information related to social relationships. The context model also takes into account the changing virtual environments that occur when users engage in online interactions and video conferencing.

\subsection{Contributions}

In this thesis, by surveying existing context-aware systems, a new dynamic user-centric mobile context model is developed and evaluated, and the model increases the expressiveness of existing context models and captures dynamic interactions among contextual elements such as tasks, roles, groups, and physical and virtual environments. The context model focuses on high level contextrelated abstractions. The model reflects the dynamic nature of context-aware systems and supports user-centric approaches. Four case studies which apply the context model to the mobile domains illustrate the usefulness and applicability of the context model.

The contributions of this thesis include the following:

- A survey of existing context aware systems;

- The proposal of a dynamic user-centric context model; and

- The presentation of four case studies in the mobile application domain that illustrate the applicability and usefulness of the approach.

Existing surveys in context-aware systems mostly focus on the architecture and application levels. The survey conducted in this work focuses on the context models, and it provides new insights into context-aware systems through a detailed look at fundamental contextual elements in context models and functionality of context-aware systems. The survey contributes a fundamental element and a 
functionality classification frameworks as well as comparisons of existing context-aware systems against the proposed classification frameworks.

The existing context models have focused more on modelling the static associations among contextual elements and system related contexts. There is a need for abstractions that model dynamic interactions between contextual elements and user context in context-aware systems. Even though online interactions are very common and popular in people's daily lives, virtual environments have not been modelled by most of the existing context models. The context model of this thesis considers dynamic interactions among contextual elements, and it focuses on three user contexts specifically roles, preferences, and social relationships. The model also incorporates virtual environments to represent the online interactions. The contributions of the context model include abstractions to model user contexts, virtual environments, and the dynamic interactions between these contextual elements.

Four case studies in different mobile application domains illustrate the usefulness and applicability of the context model. These case studies are in the domain of mobile e-healthcare, mobile commerce, mobile tourism and mobile augmented reality gaming. The case studies show the dynamic interactions modelled in the user-centric context model. They also present new types of context-aware mobile applications that provide personalized and adequate assistance to users.

\subsection{Thesis Outline}

The structure of this thesis is as following. Chapter 2 of this thesis presents the context models related to the model. Chapter 3 presents a survey of existing context-aware systems, and the survey examines the existing context models as well as the functionality provided by existing context-aware systems. Chapter 4 introduces the proposed dynamic user-centric context model with class diagrams to show the design as well as sequence diagrams to show the dynamic behaviour. Chapter 5 presents four case studies to illustrate the design further and show the potential new applications. Chapter 6 concludes this thesis and proposes future work. 


\section{Chapter 2}

\section{Related Work}

Context models represent an abstract view of contextual information in context-aware systems, and have been proposed for various domains including smart campus [25], learning [26] and pervasive services [27]. In terms of approaches taken to describe context models, the data structure used ranges from simple key-value pairs [28] to object-oriented [29, 30] and ontology-based approaches [27, 31]. This chapter presents context models that are related to specific aspects of the proposed context model. However, the proposed dynamic user-centric context model is different from the existing context models in that it considers the mobility of users and captures the dynamic interactions involving user contexts and virtual environments. The details of the context-aware systems mentioned in this chapter will be included in Chapter 3.

\subsection{Mobile Contextual Elements and Mobile Context Models}

The benefits brought by applying context-awareness to mobile application domains have motivated the research in context-aware mobile systems. However, most of the existing context models that are applied to the mobile domain are limited to their specific applications.

MIcontext [32] considers various types of contextual elements for mobile application design. Even though many types of contextual elements are proposed, this work stays at the conceptual level and details of the work are not provided. Moreover, it does not consider the associations between contextual elements. This thesis shows the details of the context model through class and sequence diagrams. Furthermore, the context model in this thesis considers the associations and the dynamic interactions between contextual elements to model the highly dynamic nature of context-aware 
systems. Each of the case studies instantiates part of the model to illustrate the usage of the model further.

SBCA [33] proposes a context ontology for mobile devices, and its interface is able to adapt to the user's current activity. However, SBCA focuses on modeling the sensor level contextual elements such as sound intensity, light intensity, and frequency of users' movements. The proposed approach in this thesis focuses on high-level user contexts, such as social relationships.

CAMob [34] is a mobile handheld system that is designed for the communication and collaboration between healthcare professionals in the hospital environment. In the hospital environment, a doctor may not know the identity of the next checkup nurse, and the next checkup nurse is known by his/her role. Thus, CAMob includes user roles to facilitate the communication and collaboration process. However, other contextual elements such as social networks and preferences are not considered. The proposed model in this thesis incorporates the role context as well as other user context elements to enable more application domains and provide more comprehensive help.

Murshid [35] is a context-aware tour guiding mobile application. One of the functions provided is allowing tourists to bookmark and share their findings with specific groups of users. According to one's interest Murshid may provide this information to the user. Even though user preferences are taken into consideration when suggesting potentially locations that the user may be interested, the recommendation module could provide more adequate suggestions by considering more contextual elements. For example, by modelling social relationships and other users, the system will be able to provide recommendations based on comments from people on the user's social networks. The proposed approach in this thesis models user context such as user preferences, roles, and social relationships to enable richer user experiences.

\subsection{Dynamic Context Models}

The context of a user is highly dynamic and is changing at every second. The change of one contextual element may propagate to other contextual elements. Even though, it is essential to model these dynamic interactions, most of the existing context models have not explicitly modelled them.

In SOCAM [36], these dynamic interactions between contextual elements are classified as dependencies between associations. Associations describe relationships between contextual elements. However, the dependencies considered by SOCAM focus mainly on sensor-based contextual 
elements such as location and weather conditions. For example, whether an activity is feasible depends on the user's current location, whether the user has the required materials, and the weather condition. Another example is that what a user is engaged in depends on his location and the status of the object with which he is interacting.

GraphicalCM [30] also considers the dependencies between associations, but the dependencies considered by GraphicalCM only relate to location context. Even though both SOCAM and GraphicalCM consider the dynamic interactions between contextual elements, they have not explicitly shown these interactions for user context.

\subsection{User-Centric Context Models}

Most context-aware systems have focused on system and location context while providing partial support for user context [37]. SOUPA [31] is an ontology designed to support pervasive applications, and it models intelligent agents and other relevant information. SOUPA includes user context such as beliefs, desires, intentions, and background information. Even though SOUPA is one of the most comprehensive context models, it does not model dynamic context changes and interactions. CoDAMoS [38] is an ontology for creating context-aware computing infrastructures, and it models user preferences and roles. However, it does not model the dynamic interactions between these elements. The approach in this thesis focuses on three user contexts, namely user preferences, roles, and social relationships as well as the dynamic interactions between these contextual elements.

In terms of modelling social relationships, COMANTO [27] and SOUPA [31] model a social relationship as a relationship involving two individuals. U-Learning [26] models social profiles that include friends and their expertise. However, social relationships should take into consideration the dynamic aspect of user interactions and include additional model elements such as location and common interests. The proposed context model considers user context, such as roles, preferences, and social relationships as well as attributes such as interest and background that are in a social relationship.

$5 \mathrm{~W} 1 \mathrm{H}$ [39-41] proposes a user-centric context model that represents contextual information through six categories, namely who, where, when, what, how and why. The user profile, preferences and tasks are modelled. In contrast to the proposed model, $5 \mathrm{~W} 1 \mathrm{H}$ does not support dynamic interactions involving roles and social relationships. 


\subsection{Modelling Virtual Environments}

Even though various forms of virtual environments have become part of people's daily lives, most context models do not consider the possibility of virtual environments and focus on modeling the physical environment instead. Examples of physical environment related properties include weather conditions, movement and light level. Virtual environment refers to an environment where objects may not have a physical presence. Online gaming, video conference, and a wireless network are examples of virtual environments.

XCM [42] defines a virtual environment as a social environment. An entity in a social environment refers to the capability of belonging to a social structure. For example, a person may belong to a soccer team. However, there is little support for online interactions and virtual environments that may be restricted by specific locations or regions. For example, a hospital network that a user can only access when he is close to or inside the hospital building is a virtual environment that is restricted to a specific region.

\subsection{Conclusion}

Most of the existing context models that are applied to mobile domains are limited to specific applications. In contrast, the approach taken in this thesis aims at providing a generic context model which takes mobility and user context into considerations, and is not limited to specific applications. Moreover, most of the existing context models have not captured the dynamic interactions between contextual elements. Some of the models that consider dependencies focus on location and sensory level contexts. The proposed approach models the dynamic interactions through dependencies between contextual elements. The context model captures the dynamic aspect of user context as well. Furthermore, most of the existing context models often focus on contextual elements related to devices and networks. However, since context-aware systems need to adapt to user context to provide better services, user context should be considered in depth. The model in this thesis includes user context, especially preferences, roles, and social relationships. Finally, even though online interactions are popular and common, the current context models focus heavily on the physical environment. The model considers both physical and virtual environments. In conclusion, the dynamic user-centric context model in this thesis is different from all the related work just described in that the model includes dynamic interactions among user context and virtual environments. 


\section{Chapter 3}

\section{Context-Aware Systems}

Most of the research efforts on surveying context-aware systems have focused on the application and architectural levels. This chapter considers context-aware systems from the perspective of higherlevel abstractions, especially in context models and the functionality of context-aware systems. Most of the existing work surveys context models in terms of their data structure, and only a few take a look at the primitives in the context models. The focus of this survey is on the identification of primitive contextual elements rather than the data structure or language that implements the model. In the rest of this chapter, the phrase fundamental element will be used to represent the primitives in context models and the word formalism will be used to represent the data structure of context models.

Context should be considered at the modelling level to avoid starting from scratch every time a system is going to be built, and other benefits include enabling the model driven approach to generate code automatically as well as reducing the implementation effort. Following the same principle of viewing context-aware systems at higher level of abstractions, this chapter surveys context-aware systems from a functional point of view. The research community has focused extensively on different architectural components in the systems. However, this chapter is more interested in a system's functionality than in its structure.

The contributions of this survey include a context model classification framework based on the fundamental elements, an illustration of how higher level context can be derived from these fundamental elements, a functionality classification framework for context-aware systems, and a comparison of existing context-aware systems against the proposed classification frameworks. The approach taken in this survey avoids having a large set of elements in the context model and provides the ability to create higher level context by combining different fundamental elements. The results of this survey suggest comprehensive fundamental element and functionality classification frameworks for context-aware systems as well as future research directions to improve the state-of-the-art of 
context-aware systems. The gaps in the existing works have motivated the development of a dynamic user-centric context model that considers mobility and dynamic interactions involving user context and virtual environments.

Part of the result in this chapter has been published in [43].

\subsection{Existing Surveys}

On surveying context-aware systems, the research community has done an extensive amount of work. Hong, Suh, and Kim [44] have classified 237 articles on context-aware systems from the architecture perspective and the research focus using their proposed context-aware system classification framework. In contrast, the focus of this survey is specifically on a classification framework for fundamental elements in context models and functionality of context-aware systems.

Krummenacher and Strang [45] evaluate ontology-based context models using context modelling criteria and ontology engineering criteria to identify the challenges and benefits of the current approaches. The context modelling criteria evaluates the applicability of the model, traceability, history, quality, inference, and incompleteness of input data. The ontology engineering criteria evaluate the reusability of the ontology, granularity of the concepts, consistency of the ontological content, and the formalism used to describe the ontology. Their work provides a classification based on the functionality and the ontology itself. However, more functionality such as context acquisition and service discovery can be studied. The authors also do not discuss the fundamental elements in the ontology.

Baldauf et al. [46] survey context-aware systems from various aspects such as architecture, resource discovery, context model, context processing, historical context data, and security to provide suggestions and insights on improving context-aware systems. In the context model section, the formalisms used by the context models are surveyed. The approach taken by Baldauf et al. focuses more on the functionality of the context-aware systems, and fundamental elements are not discussed. This survey studies the functionality in more detail by proposing more functionality such as context adaptation, and the fundamental element classification is more comprehensive because both relationships and representations of location and time are included.

While both Krummenacher et al. and Baldauf et al. focus more on the functionality of contextaware systems, Bolchini et al. [47] place a stronger emphasis on context models. Bolchini et al. 
classify context models from the data-oriented perspective by considering whether each model is able to filter irrelevant data. The context models are classified into five categories depending on the aspects that are modelled, the representation features, and context management and usage. The five categories are context as a matter of channel-device-presentation, location and environment, user activity, agreement and sharing, and selecting relevant data, functionality and services. The aspects that are modelled represent the different dimensions of context such as space, time, and user profile. The representation features are the characteristics of the formalism used such as the level of formality and flexibility. The context management and usage describe the way context is managed and used such as ambiguity and incompleteness management. However, the survey in this chapter provides more breadth by surveying more dimensions such as communication channel and environment as well as more depth by providing both relationships and representations of location and time. The result enables a more specific classification framework. In terms of the functionality of context-aware systems, this chapter surveys more functionality such as service discovery as well as privacy and security.

Ye et al. [48] evaluate the ontology-based context models based on three aspects, the proposed ontology design principles, fundamental elements and themes in pervasive computing. The goal is to identify problems of ontology-based models for future usage of these models. For context model, the previously described work by Bolchini et al. surveys only three elements, space, time and user profile, but Ye et al. include location, person/agent, time and activity. The focus of Ye et al. is the modelling of these four elements, but other dimensions such as environment are not mentioned. This survey contributes because of the wider dimensions surveyed and the ability to derive higher level context such as social relationships. From that perspective, this survey is different from all of the related work just described.

\subsection{Classification Framework}

The classification framework contains two parts: the fundamental element classification framework for the contextual elements in context models as well as the functionality classification framework for services and attributes of context-aware systems. Context models specify the relevant contextual information and the formalism used to structure the information. Context has several dimensions such as location, time, social networks, and environments, and these dimensions can be seen as 
categorizations of the fundamental elements in the context models. The fundamental element classification framework proposes the primitive elements in context models, and more complex or abstract context can be constructed from these basic primitives. The functionality classification framework proposes possible functions of context-aware systems.

\subsubsection{Fundamental element classification framework}

The fundamental element classification framework in this section categorizes the elements in the context models into five categories: entity, communication channel/network, environment, location and time. Table 1 summarizes the fundamental element classification framework.

\section{Entity}

Entities represent the parties involved in context-aware systems, and it can be a user, a device, or a service provider.

User represents the person who uses the system or application. A user profile is often used to record static or basic information about the user. User preferences can be anything from food to device and network preferences. For example, a user may prefer French food over Chinese food, and the context-aware system should take this into considerations when deciding what to suggest for dinner in another city. Some examples of preferences related to the device are the font and colour on the mobile device. User task is the activities in which a user is currently engaged. For example, the user may be dining, having meetings or sending emails.

Device represents a computational artifact interacting with a user. A device has three aspects: hardware, software and interface. The hardware represents the physical aspect of a device, and it can have features or limitations such as processing power, battery life, and memory size. The software represents the programs or applications running on the device. A device also has interfaces to interact with users through its input channel, such as a keyboard, and output channel, such as a display. 


\begin{tabular}{|c|c|c|c|c|}
\hline & & & Preference & \\
\hline & & User & Task & \\
\hline & & & Profile & \\
\hline & & & & Processing Power \\
\hline & & & Hardware & Battery Life \\
\hline & & Doxico & & Memory Size \\
\hline & Entity & Device s s & Software & \\
\hline & & & Intor & Input \\
\hline & & & Micriace & Output \\
\hline & & & Agent & \\
\hline & & Sorvico Drovidar & API & \\
\hline & & service Provider & Application & \\
\hline & & & Profile & \\
\hline & & Vi & Wi-Fi & \\
\hline & Chonnel/Aetworl. & VIIICTCSS & Bluetooth & \\
\hline Context & & Wired & Cable & \\
\hline & Fnvironment & Physical & & \\
\hline & & Virtual & & \\
\hline & & & Exact Coordinate & \\
\hline & & Representation & Region & \\
\hline & & & Symbolic & \\
\hline & Location & & Phycical & Near \\
\hline & & Relationshin & Finysical & Orientation \\
\hline & & & Snatial & Containment \\
\hline & & & Spatral & Adjacency \\
\hline & & & Exact Point & \\
\hline & & Representation & Duration & \\
\hline & Time & & Symbolic & \\
\hline & & & & Before \\
\hline & & Relationship & Temporal & After \\
\hline & & & & Overlap \\
\hline
\end{tabular}

Table 1 Fundamental element classification framework

Service provider represents an entity that provides services. A profile may be used to record the static information. In a scenario of synchronizing a patient's blood sugar level with a designated hospital, the user's device needs to synchronize the readings with the hospital server software. In this case, the hospital server software is a service provider, and the service being provided can be deciding 
whether to notify the doctor or log the data depending on the blood sugar level. A service provider can be an agent, an API (Application Programming Interface) or an application. An agent represents a computational object that acts as if it is a human with a goal and a desire to provide services. When a device is interacting with more complex systems such as trading systems or when querying information from other devices, it may be interacting with agents. In the hospital scenario, the device or user may be interacting with an API of the server software, and the API provides functions that can be used by the device. A system can also be an application.

\section{Communication channel/network}

A communication channel or network represents how entities communicate with each other. The entity can communicate through a wireless or wired network. A wireless network can be radio, Wi-Fi, Bluetooth or other methods that do not require a physical connection. The wired network is the channel which requires a physical medium for entities to communicate such as DSL or USB connection. Channels may have properties such as bandwidth and network delay.

\section{Environment}

Environment represents the surroundings of a user, and it can be either physical or virtual. Physical environment is the physical world in which the user is currently situated, and objects have a physical presence and location. A virtual environment is a world where objects do not necessarily have a physical presence. For example, a video conference does not happen in a physical space but rather a virtual environment. However, the user is sitting in a physical world. Virtual reality is another example where a person is in a virtual environment and everything the person sees does not have a physical presence.

\section{Location}

Location represents where the entity is, and it comprises the representations of and the relationships among objects or locations.

Representation is how a location can be modelled. It can be exact geographic coordinates, a region or a symbolic location. Exact coordinates are suitable for machine processing. One example of an exact coordinate is a GPS point consisting of longitude and latitude. Location can also be represented as a region such as a city or a country. In contrast to exact coordinates, symbolic representation of locations is better understood by human users. For example, a symbolic location can be Sarah's place 
or the university stadium. An address of a location is an interesting example. It has the property of both a symbolic location and an exact coordinate. For example, an address of a camping site usually does not mean much to us, but a home address can be a symbolic representation of the home's geographic location.

Relationship can be physical or spatial. The physical relationship is the relative position between entities or between location and entities such as near people or near a city. The spatial relationship is the relationship between locations such as containment and adjacency. For example, Toronto is within or contained in Canada.

\section{Time}

Time represents the temporal aspect, and it comprises the representations of and the relationships between times.

Representation is how time can be modelled, and it can be an exact point, a duration or a symbolic time. Exact point represents a very precise point in time which may not always be meaningful to humans. For example, the phone rang 5377 milliseconds ago. Duration represents a continuous segment of time. For example, the meeting lasted from 9am to 11am. A symbolic time is usually the human understandable time representation such as morning and next year. If a model can represent both the duration and the symbolic time, then a meeting which starts in the morning and finishes at noon can be represented. Moreover, the notion of "the phone rang 5377 milliseconds ago" can be represented as "the phone just rang."

Relationship is the temporal relationship. The temporal relationship describes the relationship of two points in time or two durations which can then be used to represent the temporal relationship of two activities or events. The temporal relationship can be before, after or overlap. For example, the course project meeting is after the class or the course project meeting time overlaps with the class time.

\subsubsection{Derived context}

The fundamental element classification framework enables us to derive more complex context from fundamental elements in context models. Social relationship is one of the most popular and frequent contexts being modelled. In SOCAM [36, 49] and COMANTO [27, 50], a social relationship is modelled as a person-to-person relationship. By grouping two user entities together, the fundamental 
element classification framework can also represent the relationship between them as the social relationship. Moreover, social relationships can be based on location, job, school or interest groups. For example, social network websites allow users to create and join networks based on the schools they attend. Thus, social relationships can also be represented as the relationship between users and locations, and interest groups can be represented as relationship between users and virtual environments.

In COMANTO, the agenda is characterized as belonging to one person, and the agenda includes activities. The activity is then characterized by having a start time and end time and involving a physical object, location and person. Thus, COMANTO models an agenda using activities which then involve time, physical object, location and person. By this approach, agenda can be modelled by grouping these different elements together. An activity can take place in a physical environment as well as a virtual environment. For example, if the scheduled event is happening over the Internet such as a video conference, then it will be a virtual meeting. For a virtual meeting, the physical location may not be important. In another case, several people may be having the conference meeting with another group of people. In this case, both the physical and the virtual environments matter, and the approach proposed can incorporate the change by adding more elements to it. This approach is very flexible as it supports the generation of higher level context from fundamental elements.

\subsubsection{Functionality classification framework}

The functionality classification framework classifies context-aware systems from a system's functional perspective and contains five categories: system communication, system capability, representation, service, and privacy and security. Table 2 summarizes the functionality classification framework.

\section{System communication}

System communication is concerned with how context-aware systems communicate, and includes distribution, event-based and mobile communications. This survey considers whether system components are dispersed or centralized. The system may also use synchronous or asynchronous communications among components. Context is highly dynamic in nature, and users and their devices are highly mobile. They may enter and exit the system frequently, so mobility need to be incorporated 
to provide a smooth transition. Context-aware systems should also consider the fact that users and their devices may move around within the service area.

\begin{tabular}{|c|c|c|}
\hline \multirow{13}{*}{$\begin{array}{l}\text { Context-Aware } \\
\text { System Functionality }\end{array}$} & \multirow{3}{*}{ System Communication } & Distributed \\
\hline & & Event-Based \\
\hline & & Mobile \\
\hline & \multirow{6}{*}{ System Capability } & Context Acquisition \\
\hline & & Context Retrieval \\
\hline & & Context Storage \\
\hline & & History/Logging \\
\hline & & Learning \\
\hline & & Quality Management \\
\hline & \multirow{2}{*}{ Service } & Service Adaptation \\
\hline & & Service Discovery \\
\hline & Uniform Context Representation & \\
\hline & Privacy \& Security & \\
\hline
\end{tabular}

Table 2 Functionality classification framework

\section{System capability}

System capabilities define the functions the system can perform on the parameters that describe the context.

Context acquisition: Context-aware systems may be able to capture context through sensors or through communication with other information sources. For example, a mobile device may not have GPS capability, but it may still infer a user's location based on the user's calendar or other GPSenabled devices in the neighborhood.

Context retrieval and context storage: Once the system gathers context-related information, the survey examines whether that system provides the functions of context retrieval and context storage. Context retrieval allows users or other systems to query the collected contextual information, and context storage refers to the ability to store the collected contextual information.

History/logging, reasoning, and learning: Simply storing the contextual information is not enough to support smarter services so the survey examines a system's ability to process contextual data. The processes include logging, reasoning, and learning. History/logging stores previous actions taken by users which can be logged for future use or user habit analysis. Reasoning is concerned with whether the system is able to use the collected contextual information to map low-level information to 
symbolic contextual information or to deduce higher level information. An example of the former type of reasoning is mapping an exact coordinate to a symbolic location such as an address. An example of the latter is that the system may be able to determine that the user is in a room where a presentation is in progress so the cell phone should be switched to silent mode. Based on the logged data or reasoning, the system may be able to learn. For example, whenever the user enters a room which has a meeting in progress or where the next event is a meeting, the user turns the volume of the cell phone down. The system may learn the behavior and reduce the volume automatically for the user.

Quality management: Depending on how the context is captured, the information might be incomplete, ambiguous, conflicting or missing. In these cases, the system should have basic quality management to be stable and perform robustly.

\section{Service adaptation and discovery}

This survey examines whether the service provided to users can adapt to different situations. For example, the device may adjust the screen brightness, font size or color depending on the room lighting or the screen size of the device. This survey also examines whether the context-aware system advertises its services so a new device coming into the service area can discover what type of services or resources are available.

\section{Uniform context representation}

Significant effort is needed to be expended on integration so that systems work seamlessly together. The survey examines if the system provides a uniform knowledge representation scheme such as an ontology to describe the contextual information so several systems can easily communicate.

\section{Privacy and security}

As the system gets smarter, a larger amount of user data is needed to create tailored services. However, users need to have control over what information is shared and with whom including service providers and how long the information should be kept. This survey examines if the system provides privacy and security options for the users so they have control over their information. 


\subsection{Context-aware System Classification}

In total, 22 context-aware systems are surveyed, and the survey compares them against the classification frameworks to gain more insight into the state-of-the-art of context-aware systems.

\subsubsection{Context-aware systems}

This section first introduces the systems classified by giving their system descriptions and stating their target problems. Some of the systems were not named by the original authors and this survey describes them as: ConceptualCM [51], GraphicalCM [30], SBCA [33, 52], MIContext[32] and CAMob [34].

\section{$\operatorname{CSCP}[53]$}

The aim of the Comprehensive Structured Context Profiles (CSCP) is to provide a common format for representing contextual information. The context is represented as session profiles which model device, network and user information.

\section{SOCAM [36, 49]/CONON [54]}

The Service-Oriented Context-Aware Middleware (SOCAM) architecture is developed to build context-aware systems quickly, and it aims to provide efficient context acquisition, discovery, interpretation and access. SOCAM proposes a dual-layer ontology inspired by CONON. The upper ontology defines high-level abstractions such as location, person and activity. The lower ontology defines domain-specific abstractions such as TV, food preference of a person, and entry in an indoor space. The domain specific ontology can be dynamically loaded and swapped to reduce the processing overhead. In this approach, the required context knowledge is reduced to the upper ontology, and the domain-specific ontology can be dynamically bound. The SOCAM architecture can translate sensed context to higher abstractions, and it provides functions such as interpretation, storage, and location services. The classification results are based on both the generic ontology and the sample domain specific ontology.

COMANTO [27, 50]

The COntext MAnagement oNTOlogy (COMANTO) strives to be generic and supports the sharing, synchronization, and collaboration of context knowledge. The context ontology defines abstractions 
such as person, place, preference, service and time. The Context Management System (CMS) is a middleware which makes use of the DAIDALOS pervasive service platform and COMANTO to enable context acquisition, storage, management and dissemination.

CoBrA [25]

The Context Broker Architecture ( $\mathrm{CoBrA})$ is an agent-based infrastructure that aims to help agents acquire, reason, and share context knowledge in a smart meeting room system on a university campus. The CoBrA Ontology describes the following four concepts and the properties among them: places, agents, agent location context and agent activity context. Places represent the physical locations on a university campus, and agents represent software or human agents. Agent location context describes the location of an agent. Agent activity context describes the activities the agent participants. The context broker is the center of the architecture, and it manages a shared context model among other components in the architecture. They are the context knowledge base, context reasoning engine, context acquisition module, and privacy management module.

\section{SOUPA [31]}

Standard Ontology for Ubiquitous and Pervasive Applications (SOUPA) is designed to support knowledge sharing and context reasoning as well as to achieve interoperability in ubiquitous and pervasive applications. This ontology includes vocabulary representing an intelligent agent's context such as its beliefs, desires and intentions in the ubiquitous world. SOUPA ontology consists of the SOUPA Core and the SOUPA Extension. The SOUPA Core specifies the generic vocabulary such as agent, time and space. The SOUPA Extension defines the domain specific vocabulary such as documents and location.

\section{U-Learning [26]}

U-Learning proposes a context model to describe and acquire contextual information specifically for a Web learning-environment. The model defines context from both the learner and the learning content's perspectives. For the learner, context is the environment that affects how the learner discovers and accesses Web learning content. The learner ontology defines learner profile, preference, Quality of Web Services profile (QoWS), and environment. From the perspective of the learning content, context is the surrounding that affects the delivery and presentation of Web content. In the 
content ontology, four different types of metadata are defined to support semantic representation at different levels.

\section{ASC $[5,55]$}

The Aspect-Scale-Context (ASC) model is a very general way to view context. It defines three core concepts: aspect, scale and contextual information. Aspect can be seen as one category or domain of contextual information, scale as one dimension or measurement unit, and the contextual information as the real value of the context. For example, if the aspect is spatial distance, then scale can be meter or kilometer and contextual information can be 10. The ASC model also defines IntraOperation and InterOperations for translating between scales in the same or different aspects. A MetricOperation allows comparison between contextual information based on implementation details. The classification is based on the sample basic aspect, scale, and contextual information provided. Strang et al. [5] apply the ASC model to Web services to describe facts and relationships, and they focus more on the context-provider domain in the sample application. The ASC model is described using the Context Ontology Language (CoOL) which combines three ontology languages: OWL, DAML+OIL, and F-Logic. By using several ontology languages, the developers can choose the language that is most natural to their applications.

\section{GLOSS [56, 57]}

The GLObal Smart Space (GLOSS) provides location-aware services to support the interactions among people, artifacts and places on both the global and local scales. "Where" is used to define places and any geo-spatial concepts. The location-aware services include the detection, delivery, storage and exploitation of location information. On the global scale, the architecture makes use of peer-to-peer architecture with hierarchical nodes. Pipelines are used for local event delivery. GLOSS is different from other systems surveyed in that its focus is on location context only.

\section{MAIS [58]}

The Multichannel Adaptive Information Systems (MAIS) project studies adaptive information systems at all levels from application to network and device. The MAIS architecture aims at supporting adaptation at all levels for distributed systems. This survey will only classify the elements in the reference model which are designed to be used during e-service invocation because the details are not discussed fully in the paper referenced. The reference model considers e-services from the 
perspectives of service provision and actor context. The service-provision perspective describes the functions of e-services such as the description of service provider and the functions provided. The actor context describes the user characteristics such as preferences and expertise.

HyperAudio [4, 59]

HyperAudio is a location-aware adaptive-system designed to be a museum guide, and it can adapt to individual visitors based on their profile, preferences and locations in the museum. It has the knowledge of the physical organization of the museum. It can also interpret users' interactions, record the history of the users' locations and information, and decide on the content to present. HyperAudio considers the location context and the visitor's background information to provide personalized services.

\section{ConceptualCM [51]}

ConceptualCM is a framework which provides an ontological foundation model and a runtimeinfrastructure model. In the ontological foundation, context as a whole is viewed as a process and is modelled as acyclic state graphs. Each node denotes one particular context and the edge denotes a condition of context change. Each context is characterized by a set of entities, roles assigned to entities, relationships between entities, and situations. The runtime-infrastructure model provides context-aware system-architecture for supporting essential services, such as sensing, providing symbolic observables, communicating between application and infrastructure, privacy, and trust. Moreover, the authors comment that system adaptation and context acquisition are essential to providing useful and usable services.

\section{CoDAMoS $[38,60]$}

The Context-Driven Adaptation of Mobile Service (CoDAMoS) project proposes an extensible context ontology which aims at addressing challenging issues, such as application adaptation, mobility, service discovery, and content adaptation in ambient intelligence. The ontology describes four basic entities: user, environment, platform, and service.

\section{GraphicalCM [30]}

GraphicalCM is an object-based context model. The main concepts of the model are entities, attributes, and the associations between entities. Entities can be physical or conceptual objects such as 
a person or communication channel. Entities have attributes such as the name of a person or the identifier of a communication channel. The associations are the links from one entity to its properties or other entities. An example is the entity 'person' located near the entity 'device'. GraphicalCM also distinguishes between different types of associations, and it models dependency and context quality. The sample scenario is built around people, communication devices and communication channels.

\section{SBCA $[33,52]$}

The mobile device sensor-based context awareness (SBCA) framework enables real-time context recognition in an uncertain environment. It also supports the development of context-aware mobile applications by abstracting sensor inputs to a higher-level context. The ontology in the framework focuses on the contextual information gathered by sensors such as physical environment, device position, and the user's movement. The framework is able to recognize high-level context using a naïve Bayes classifier even with uncertain and noisy contextual information input. The sample application is able to change the font size, screen brightness and content based on user activity and light level in the surroundings.

\section{OwISrv [61]}

OwlSrv is a prototype context-aware system that provides storage, inference and query of contextual information. A Web based calendar is provided as the client application. The ontology proposed focuses more on location modelling. The authors propose to model social relationship and schedules. The work seems to be at an early stage, and the paper referenced presents the experimental work.

\section{Perceptual components [62]}

The ontology is derived from what the system is able to sense and process as well as user tasks. Context is viewed from both system and user perspectives. User context is characterized by roles and relations. The key for the design of the architecture is that context-aware systems need to be able to sense the surroundings. The system gets data from the low-level sensors. The process in the system that transforms or interprets the sensory data is called an observational process. The architecture is based on a dynamically assembled federation of observational processes. This architecture allows the system to recognize entities such as eyes or faces from the observables. 


\section{GAS [63]}

The Gadgetware Architectural Style (GAS) Ontology is designed to provide semantic interoperability for heterogeneous devices, support the dynamic nature of ubiquitous computing environments, and provide service discovery for devices. The GAS Ontology has two layers, namely the Core Ontology (GAS-CO) and the Higher Ontology (GAS-HO). The GAS-CO describes the basic concepts of ubiquitous computing environments and the inter-relations between heterogeneous devices to enable communication and collaboration among these devices. The GAS-CO describes smart devices at home, and they are modelled by the physical characteristics (TPlug), their capabilities (SPlug), the communication channel (synapse), and what signal the devices can transmit and perceive (service). The GAS-HO describes a specific instance, such as a smart lamp, and it contains the dynamic information acquired by the instance through its usage and interactions with other smart devices.

\section{XCM [42]}

$\mathrm{XCM}$ focuses on the coordination of context, and the computation is a separate concern not presented. The model is based on four concepts: entities, environments, social laws, and ports. The entity can be anything that could be coordinated. It can be composed of other entities, and the compound entity is the environment of the inner entities. The social laws determine how the entities can be composed, and the port represents the communication channel between entities. In this work, the authors try to be general, and the entity ontology only defines the structure such as "HasComponents." Moreover, the entity can be anything. Communication can be seen as an agent coupled with a port, and the port is the communication channel for the agent. The coupling can be defined by the social law because port and agent are seen as entities.

\section{MIContext [32]}

MIContext is a context model for mobile interactions. Many contextual elements for mobile interactions are proposed to highlight potential new ways to assist users through mobile devices. The goal is to reexamine the design of mobile devices to take advantage of the fact that these devices are always carried by users. Some of the main categories of contextual elements that are taken into consideration include device, communication channel, interface, user tasks, activities, environment and culture. MIContext focuses on the conceptual level only. 


\section{CAMob [34]}

CAMob is a context-aware handheld system designed for communication and collaboration in the hospital environment. The system makes use of the agent-based architecture, and the communication and collaboration between users through their devices are managed by the context-aware client and the instant messaging server. Communication in the hospital environment requires more than location and identity of the users. CAMob thus considers location, time, role, and artifact state as the four main contextual elements. The location is used to determine the most relevant help and information that the system should provide to the healthcare professionals. By modeling the role context, CAMob allows healthcare professionals to collaborate based on their roles since they may not know the identity of their collaborators.

\section{Murshid [35]}

Murshid is a context-aware tour guiding mobile application proposed for tourists in the United Arab Emirates (UAE). The application analyzes different contextual elements to adapt its functionality to the users' surroundings. The context model deployed considers user location, user profile, current date, UAE event information, and user's interaction with the device. The user profile records users' background information. UAE event information records the details of events that take place in UAE. Based on these contextual elements, Murshid provides five types of services to the mobile device users. These services are special attraction event notifications, weather forecasting, currency exchange rates, language translation, and location bookmarking and sharing. Tourists can share interesting findings with specific groups of users, and according to one's interest Murshid may provide this information to the user.

\section{$5 \mathrm{~W} 1 \mathrm{H}[39-41]$}

$5 \mathrm{~W} 1 \mathrm{H}$ proposes a user-centric context model to represent user context for sharing context information among services efficiently. It brings a unified view of user context through six categories. They are user's identification (who), user's location (where), time frame that the context is available (when), the object user is paying attention to (what), user's behaviour (how), and user's intention (why). Contextual information is classified based on the source of information and the subject that exploits the information. Preliminary context represents pieces of data received from various sensors. Integrated context represents processed and reasoned contextual information that fuses with various preliminary contexts. Conditional context is specified by the user to describe the conditions that 
should trigger the desired services. Final context interprets both integrated context and conditional context to find matches and trigger a service.

\subsubsection{Results of the fundamental element classification}

This section compares the context models surveyed to the classification framework, and Table 3 summarizes the results of the fundamental element classification. The highlights and comparisons of some of the results will be presented in the rest of this section. The context models are surveyed by referencing the relevant papers found in the literature. A dimension, such as location and time, is modelled if the paper referenced explicitly indicated that it is modelled. For the cell containing '*, it means that the authors imply that the particular contextual element is modelled. For the cell containing '**,' it means that the authors did not give enough detail on the modelling of specific attributes. For service provider and communication cannel/network elements, this survey does not relate them to the specific sub-categories in the result.

\section{Structure of context models}

Most models use ontologies to represent contexts. Three types of the approach can be identified: domain specific, remembering all, and layered. OwlSrv considers a domain and creates a specific ontology. COMANTO provides a very general ontology which tries to capture all the possible domains of contextual information. However, this approach may require a large amount of storage for the context knowledge. On the other hand, SOCAM, SOUPA and GAS all take the layered approach. They employ both a higher ontology and a lower ontology. The higher ontology describes the abstract and general concepts, and the lower ontology describes the domain-specific context. The layered approach is more space efficient. SOCAM claims that the domain-specific ontology can be loaded and re-bound dynamically so a large storage space to remember all knowledge is not necessary. Moreover, the layered approach can easily incorporate new context that was not modelled in the original domain-specific ontology. 


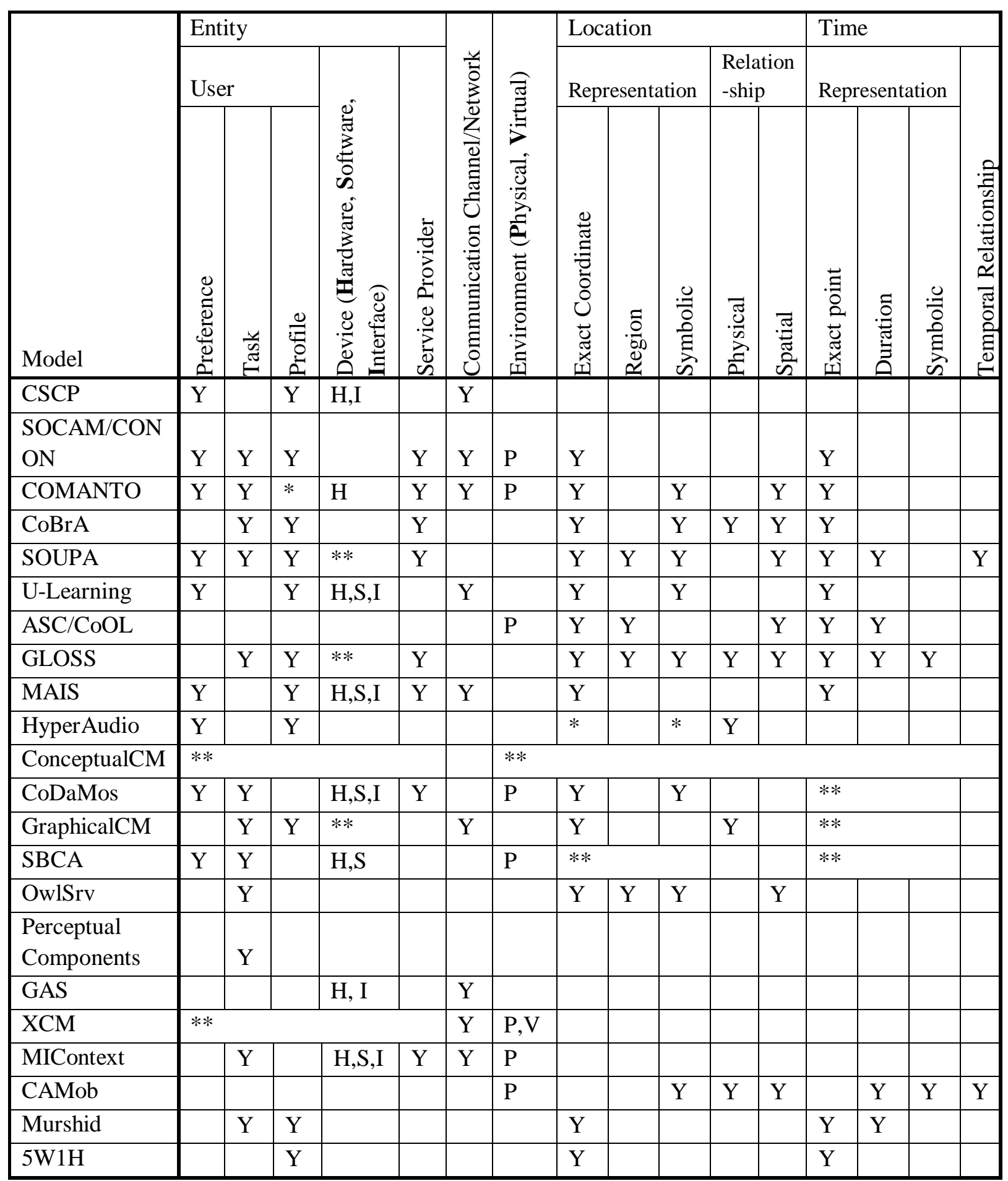

* implicitly indicated, ** not enough details

Table 3 Result of the fundamental element classification 


\section{System perspectives versus user perspectives}

Some models such as GAS, perceptual component and SBCA, consider context from the system perspective. They explicitly consider sensory data gathered from sensors or devices. GAS considers

what a sensor of a device is capable of transmitting and perceiving. The context considered by GAS and SBCA is at a lower level and closer to raw sensory data. On the other hand, U-Learning and HyperAudio consider context from the user's perspective, and they consider higher-level information sources such as calendars and users' previous experience in visiting a museum.

\section{Granularity of elements}

The context models differ not only in the fundamental elements modelled but also on the granularity of the elements. On one side, SOCAM models TV and DVD players and tries to be very detailed and specific. On the other side, XCM takes a very general approach and models everything including devices as entities. GLOSS also models devices in a very general way, and it defines the concept of instrument which refers to an object that a person can hold and operate.

\section{Fundamental elements modelled}

People, device, location and time are the most common fundamental elements among the context models surveyed. For modelling the environment, only XCM has considered the possibility of a virtual environment, even though context will be of consideration in future virtual reality and augmented reality applications. Moreover, online interactions such as online chat room and video gaming are already very popular. For location modelling, GLOSS is a location-based system and has the most comprehensive location model among all the context models surveyed. It covers all the proposed ways to represent location information and relationships between locations and people.

\section{Modelling dynamic interactions}

The relationships between fundamental elements have not been modelled extensively. Especially, the aspect that a change in one fundamental element may initiate changes in other contexts is not modelled by most of the systems surveyed. Composition, ownership and other static relationships are common in the models surveyed. For example, CoDAMos models hasProfile and useServices relationship, and COMANTO models including and locatedIn relationship. Only SOCAM and GraphicalCM consider the dynamic interactions between the fundamental elements. Both of them 
model the dynamic interactions through dependencies between the associations that describe relationships between contextual elements.

\section{Conclusion with respect to the fundamental element classification}

None of the models include all the dimensions proposed in the fundamental element classification framework. Most models are still application specific, and they focus on one particular domain or scenario. However, some research projects are trying to be general. SOCAM and SOUPA propose general ontologies to be applied beyond the sample application domains. ASC proposes to view context as aspects, and it is described using three different languages so as to incorporate the benefits of all of them and provide the ability to select the most suitable ones at all time. There is still a need for comprehensive meta-model or model general enough that will support all the elements proposed for context modelling.

\subsubsection{Results of the functionality classification}

This section presents the result of the comparison of context-aware systems to the functionality classification framework, and Table 4 summarizes the results of the functionality classification. The rest of this section presents the highlights and comparisons of some of the survey results. A function is indicated as supported if the paper referenced has explicitly mentioned it. The resulting table is quite sparse as most context-aware systems have specific target application domains. The papers referenced may not have explicitly indicated all the functionality the system supports.

\section{Aiding the dynamic nature of context-aware systems}

A context-aware system is highly dynamic because of the dynamic nature of context. At any point, the time is ticking, and context changes. Users and devices may enter and leave the service area frequently. Service discovery and mobility should be a big concern to provide smooth transition from one environment to another, but not all systems have considered this aspect. Events aid the communication between context-aware systems and users, but most systems do not explicitly indicate whether the system supports event-based interactions.

\section{Context processing and handling}

Most applications have the reasoning capability, and this function may include mapping low-level context to high-level context and deriving new context. However, learning and logging user 
interactions are not well supported in the context-aware systems surveyed. In terms of handling possibly error prone context input, some systems such as ASC and SOCAM choose to support quality management at the modelling level. Others such as SBCA employ statistical calculations to support reasoning in noisy environments to provide robust behavior.

\begin{tabular}{|c|c|c|c|c|c|c|c|c|c|c|c|c|c|c|}
\hline \multirow[b]{2}{*}{ Model } & \multicolumn{3}{|c|}{$\begin{array}{c}\text { System } \\
\text { Communication }\end{array}$} & \multicolumn{7}{|c|}{ System Capability } & \multicolumn{2}{|c|}{ Service } & \multirow[b]{2}{*}{ 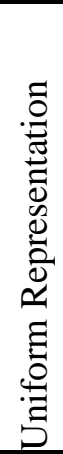 } & \multirow[b]{2}{*}{ 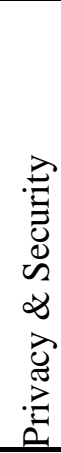 } \\
\hline & 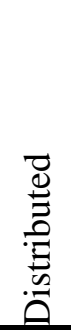 & 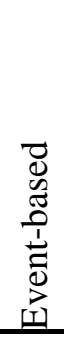 & $\frac{0}{\frac{0}{0}}$ & 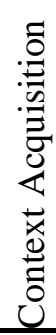 & 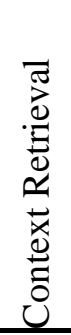 & 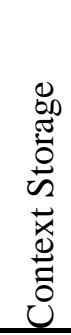 & 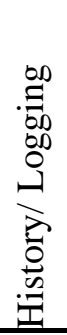 & 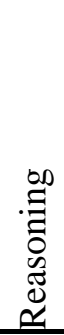 & . & 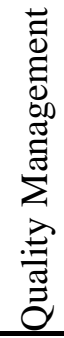 & 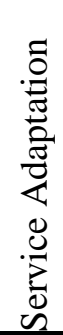 & 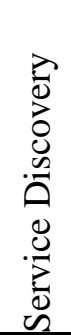 & & \\
\hline $\mathrm{CSCP}$ & & & & & & & & & & & & & $\mathrm{Y}$ & \\
\hline $\begin{array}{l}\text { SOCAM/CON } \\
\text { ON }\end{array}$ & $\mathrm{Y}$ & & & $\mathrm{Y}$ & & $\mathrm{Y}$ & & $\mathrm{Y}$ & & $\mathrm{Y}$ & $\mathrm{Y}$ & $\mathrm{Y}$ & & \\
\hline COMANTO & & $\mathrm{Y}$ & & $\mathrm{Y}$ & $\mathrm{Y}$ & $\mathrm{Y}$ & & $\mathrm{Y}$ & & $\mathrm{Y}$ & & & $\mathrm{Y}$ & \\
\hline $\mathrm{CoBrA}$ & $\mathrm{Y}$ & & $\mathrm{Y}$ & $\mathrm{Y}$ & & $\mathrm{Y}$ & & $\mathrm{Y}$ & & $\mathrm{Y}$ & & & $\mathrm{Y}$ & $\mathrm{Y}$ \\
\hline SOUPA & & & & & & & & & & & & & & $\mathrm{Y}$ \\
\hline U-Learning & & & & $\mathrm{Y}$ & & & & & & & & & & $\mathrm{Y}$ \\
\hline ASC/CoOL & & $\mathrm{Y}$ & & $\mathrm{Y}$ & & & & $\mathrm{Y}$ & & $\mathrm{Y}$ & & & $\mathrm{Y}$ & \\
\hline \multicolumn{15}{|l|}{ GLOSS } \\
\hline MAIS & $\mathrm{Y}$ & & & & & & & & & & $\mathrm{Y}$ & & & \\
\hline HyperAudio & & & & & & & $\mathrm{Y}$ & $\mathrm{Y}$ & & & $\mathrm{Y}$ & & & \\
\hline ConceptualCM & & & & & & & $\mathrm{Y}$ & $\mathrm{Y}$ & & & $\mathrm{Y}$ & $\mathrm{Y}$ & & $\mathrm{Y}$ \\
\hline CoDaMos & & & & & & & & & & $\mathrm{Y}$ & $\mathrm{Y}$ & $\mathrm{Y}$ & & \\
\hline GraphicalCM & & & & & & & & & & $\mathrm{Y}$ & & & & \\
\hline SBCA & & $\mathrm{Y}$ & & $\mathrm{Y}$ & $\mathrm{Y}$ & & & $\mathrm{Y}$ & & $\mathrm{Y}$ & $\mathrm{Y}$ & & $\mathrm{Y}$ & $\mathrm{Y}$ \\
\hline OwlSrv & & $\mathrm{Y}$ & & & $\mathrm{Y}$ & & & $\mathrm{Y}$ & & & & & & \\
\hline GAS & & & $\mathrm{Y}$ & & & & & & & & & $\mathrm{Y}$ & $\mathrm{Y}$ & \\
\hline $\mathrm{XCM}$ & & & & & & & & $\mathrm{Y}$ & & & & & $\mathrm{Y}$ & \\
\hline \multicolumn{15}{|l|}{ MIContext } \\
\hline CAMob & & & & $\mathrm{Y}$ & & & & $\mathrm{Y}$ & & & & & & \\
\hline Murshid & & & & $\mathrm{Y}$ & $\mathrm{Y}$ & $\mathrm{Y}$ & & $\mathrm{Y}$ & & & & & & \\
\hline $5 \mathrm{~W} 1 \mathrm{H}$ & & & & $\mathrm{Y}$ & & & & $\mathrm{Y}$ & & & & & & \\
\hline
\end{tabular}

Table 4 Result of the functionality classification 


\section{Security and privacy}

Security and privacy do not seem to be a big concern yet, but some systems have considered them. SOUPA supports security and privacy at the modelling level through policy, and users can specify security and privacy policies, such as who has the right to perform specified actions. On the system level, U-Learning allows users to define privacy preferences, and SBCA checks the trustworthiness of incoming context by deploying a security module.

\section{Conclusion with respect to the functionality classification}

Most context-aware systems have specific goals, and it is time consuming and really difficult to build a system that can be applied to all domains and has all the proposed attributes. However, through integration of systems, context-aware systems that serve in all domains may be achievable. Thus, the uniform representation of the context is essential to enable integration, and most models have done so through the use of ontology.

\subsection{Conclusion}

This chapter has presented the existing context-aware systems in terms of context models deployed and functionality provided, and has proposed classification frameworks for the fundamental elements in context models and the functionality of context-aware systems. The fundamental element classification framework identified primitive elements in the context models, and illustrated how the combination of these primitives can create higher-level context. The functionality classification framework proposed possible functions to be supported by context-aware systems and covered the basic functionality provided by current context-aware systems. Finally, this survey classified contextaware systems based on their context models and functionality as described in the classification frameworks. This chapter also highlights and compares some of the interesting approaches taken by the systems and potential points of improvement.

The current context models are limited to their target application domains, but the layered context model, which includes a core and an expandable domain-specific model, has the potential to incorporate new context easily. None of the surveyed context models consider all the fundamental elements proposed in the fundamental element classification framework. Only one context model considers the virtual environment and most models consider context from a system perspective. Most of the models do not consider the dynamic interactions between fundamental elements. 
The functionality of the context-aware systems is really limited to their target application domains as well. None of the context-aware systems have all the functions proposed in the functionality classification framework. Mobility was not considered by many systems even though several contextaware systems model the physical environment. This survey identified the benefits each system provides and the disadvantages of some approaches. The result identifies the gaps in the existing work and enables the proposals to advance the state-of-the-art in the context-aware systems. 


\section{Chapter 4}

\section{A Dynamic User-Centric Mobile}

\section{Context Model}

\subsection{Requirements and Overall Design}

Context changes frequently, but current context models do not focus on the dynamic interactions that result from those changes. A context-aware system needs to be able to adapt according to its user's context and assist the user with his current activities. Current context models lack support for representing these dynamic interactions, particularly those involving changes in user context and virtual environments. The goal of this thesis is to provide a user-centric context model that supports dynamic context element interactions and virtual environments [64].

The basis of the context model is the user. The user represents a person as well as his context. The model considers three main types of user context. They are roles, preferences, and social relationships. A user has profiles which define information related to the user in different situations such as a professional profile and a gaming profile. Each profile may include demographic information, potential roles, and potential preferences. A preference describes a user's desire for different services or device behaviours in different situations. A user has a social relationship with another user, and a social relationship is more than just a relationship involving two individuals. It may include other elements such as location, preferences and roles. A group may consist of users and a user may be in a group at one point in time. A user is in a physical environment but may or may not be in a virtual environment. Figure 1 shows the overall design of the context model. 


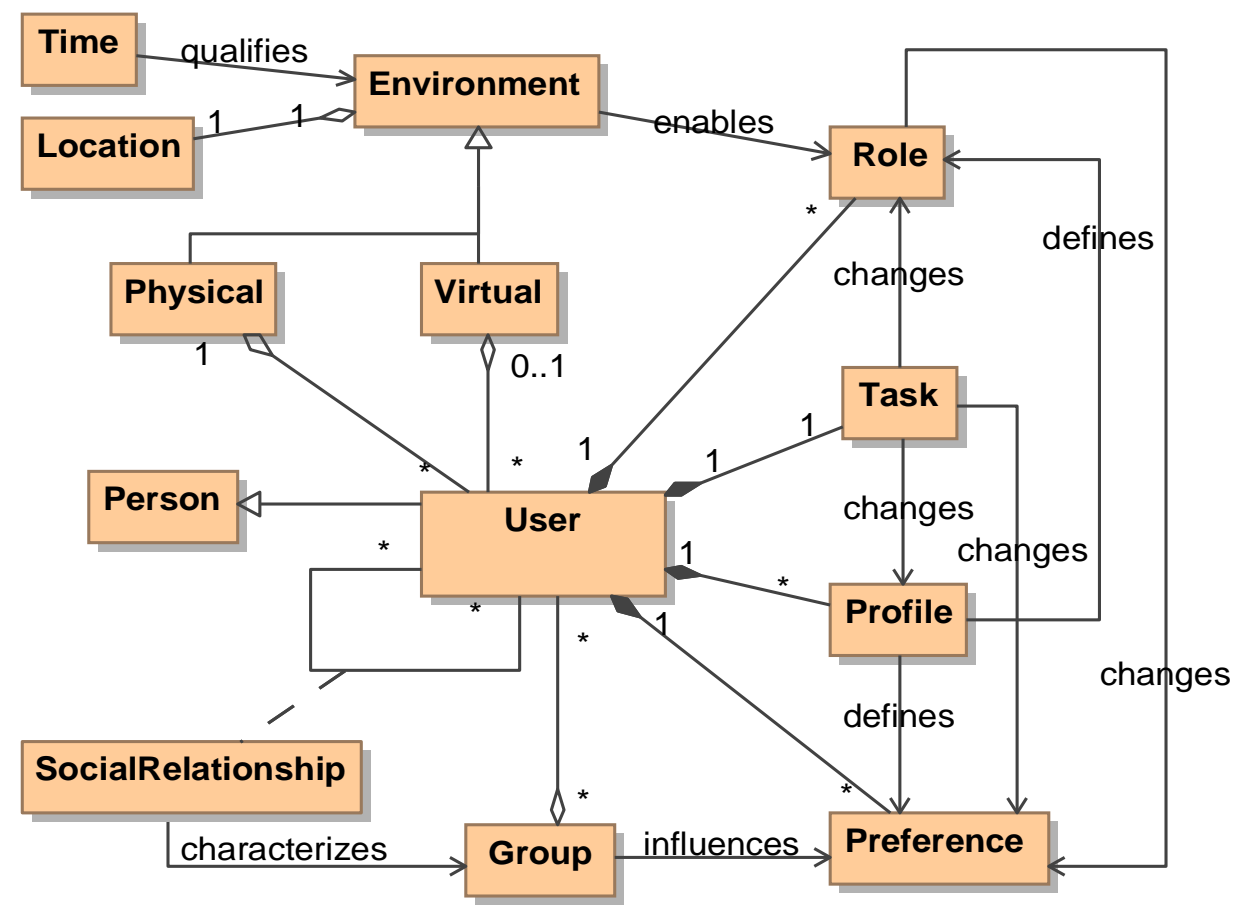

Figure 1 Overall design

The model also supports the relationships between a user's context elements. These relationships are dependencies present between context elements. These dependencies allow the context elements to respond to changes in other context elements. This capability reflects the dynamic nature of a user's context. As a user's context changes, the model is able to represent those changes based on the relationships between different context elements.

For example, a user may enter a new environment and this change triggers a change in the user's context, enabling a new role. This change may result in further changes in other context elements such as tasks, profiles and preferences. A new role may enable new tasks that are available to the user. A profile may add a new set of preferences to the existing set of preferences.

The following subsections present different views of the context model through class diagrams as well as the dynamic aspect of the model through sequence diagrams. Each view shows a different aspect of the context model, namely the user view, the preference view, the role view, the task and profile view, the social relationship view, and the environment view. 


\subsection{Views}

\subsubsection{User view}

A user is a person that possesses a handheld device capable of providing context-aware services. A person has a set of preferences, defines profiles, engages in tasks, plays a set of roles, and is in a physical environment. A user inherits these properties from a person. In addition, a user may be in a virtual environment, may belong to a group, may be around a group of other users, and have social relationships with other users. He can update his profiles which may trigger changes to other contextual elements. Figure 2 shows the details of the user class and its relationship with other classes.

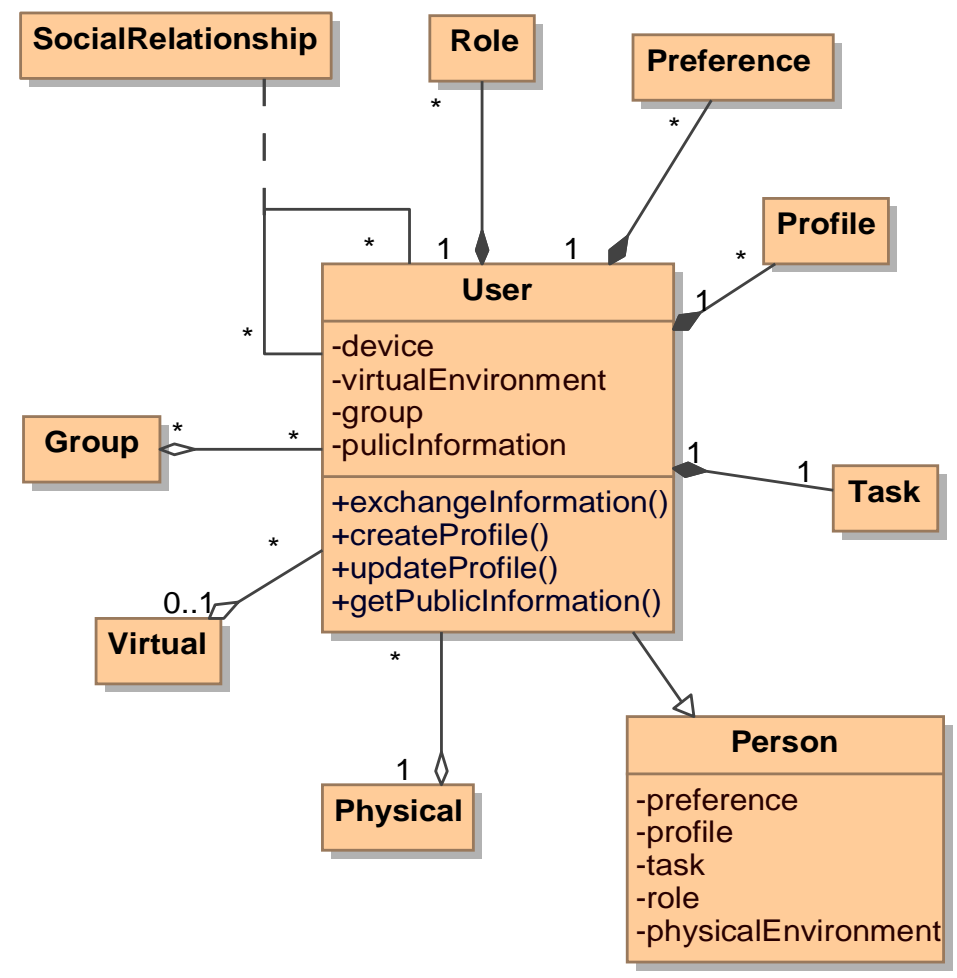

Figure 2 User view 


\subsubsection{Preference view}

A preference describes a user's desires for different services or device behaviours in different situations. A preference may include anything such as shopping recommendations and font sizes on mobile devices. A profile may define sets of preferences related to a situation. One set of the preferences would be the dominant set at one point in time. The dominant set of preferences depends on the profile, task, the relevant group of users, and role. The user may configure or update the profile directly to update his preferences. A change in task, other users around or role may also require the dominant set of preferences to be updated. Figure 3 shows a detailed view of the preference element.

\subsubsection{Role view}

A role represents one of the identities played by a user. A profile may define roles related to a situation. One of the roles would be the dominant one at one point in time. The dominant role depends on the profile, environments and the task. The user may configure or update the profile directly to update his roles. A change in the environment or task triggers a change in role. A role change may further trigger changes in the preference element. Figure 4 shows a detailed view of the role element.

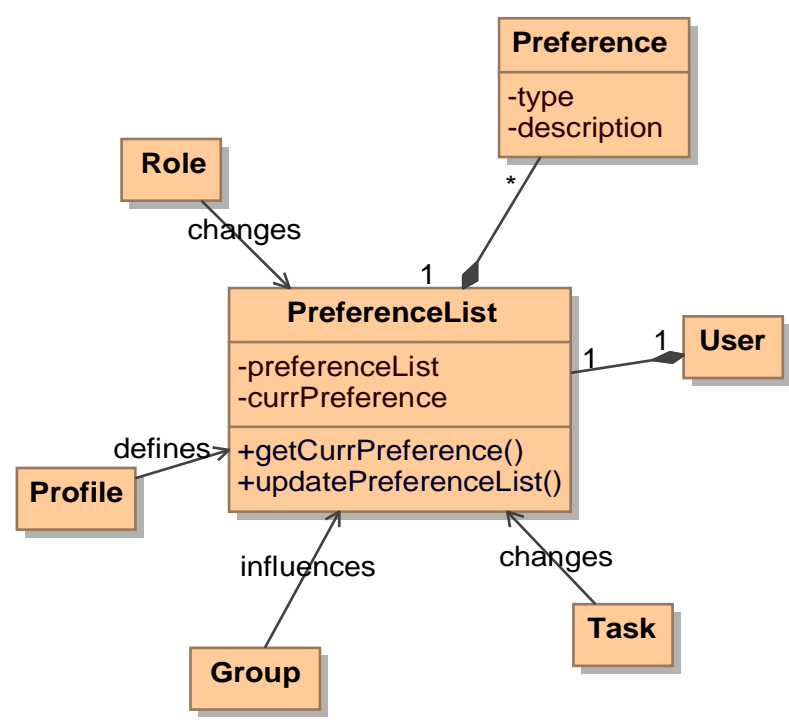

Figure 3 Preference view 


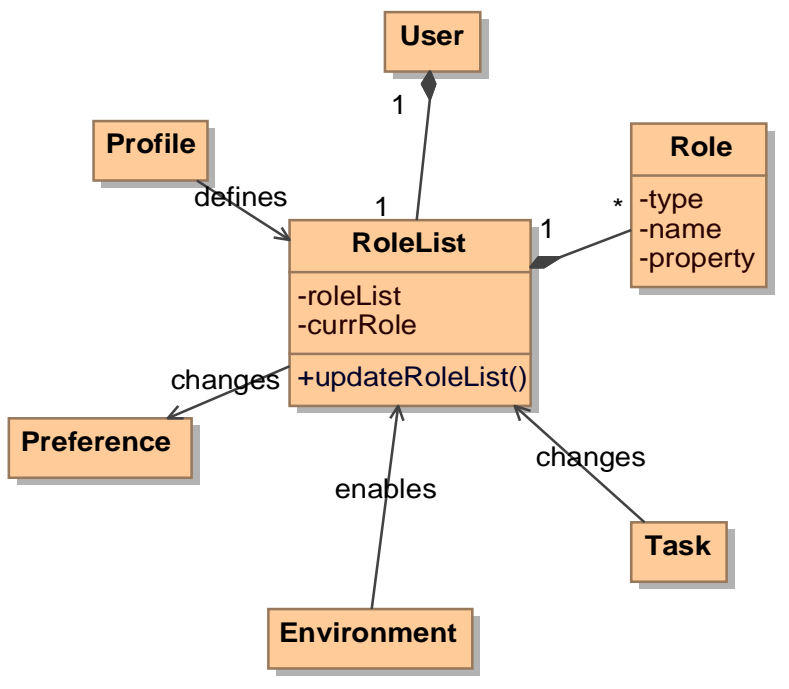

Figure 4 Role view

\subsubsection{Task and profile view}

A task describes the user's current activity. When a change in task takes place, it triggers further changes in the profile, role, and preference elements.

A user has profiles, and each profile has information related to the user in a situation. For example, the user may define a professional profile and a gaming profile. Each profile may include demographic information, preferences and roles. He can also define dynamic preferences such as a desire for the mobile device to run in the professional mode when he is around colleagues. He may configure his profile to update his preferences and roles related to the situation. Figure 5 shows a detailed view of the task and the profile.

\subsubsection{Social relationship view}

A social relationship exists between two users. A social relationship may involve common attributes such as location (e.g., from where you know this person), organization, role (e.g., teacher-student association), and preferences to describe more information about the relationship. A user may belong to a group. A group consists of users who have the same type of social relationship with a given user. The change in the group around this given user may trigger changes in this user's preferences. The group of other users can be physically around the user or in the given user's social networks. Figure 6 shows a detailed view of social relationship and group. 


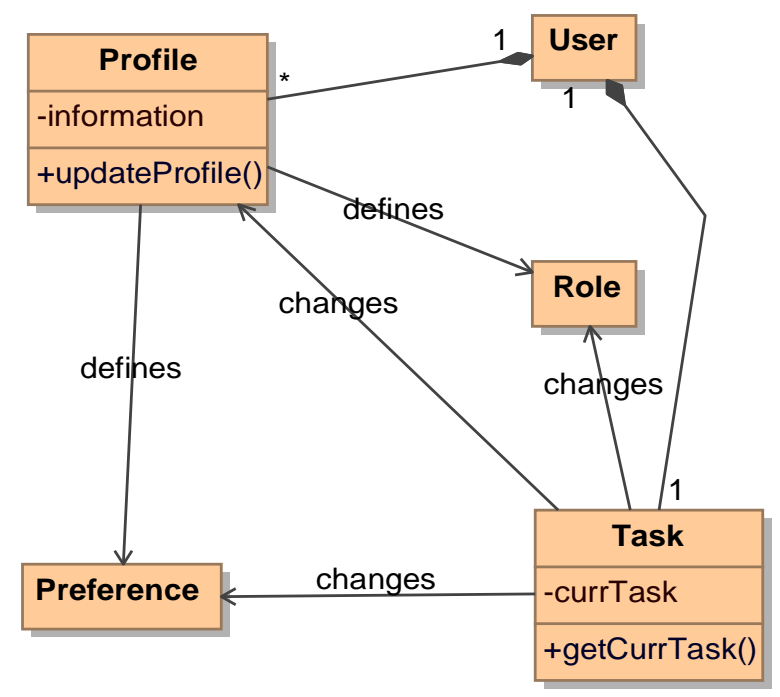

Figure 5 Task and profile view

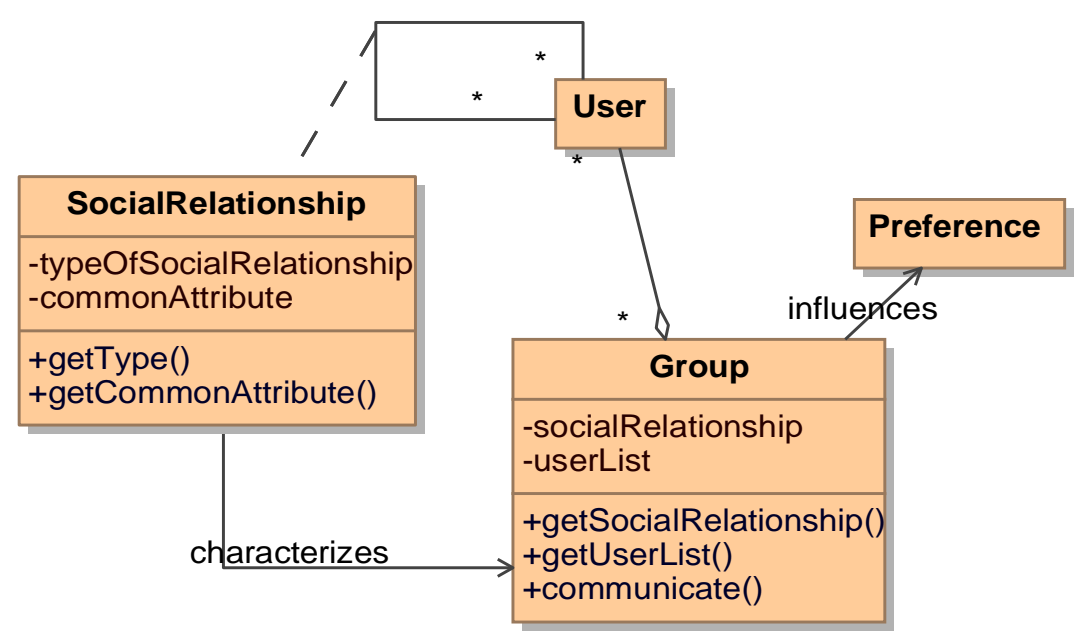

Figure 6 Social relationship view

\subsubsection{Environment view}

The model supports both physical and virtual environments. A physical environment refers to the world where an object would have a physical presence. A virtual environment refers to a world where an object may not have a physical presence. Every environment is associated with a location. A virtual environment can have a virtual location such as an URL or a location in a game. The model 
also supports a virtual environment that may be restricted to specific locations and regions. For example, a hospital network is a virtual environment that is restricted by physical region because a user must be in the proximity of the hospital to be eligible to enter this virtual environment. Time can qualify an environment. For example, a room is only qualified to be a meeting room at the meeting time.

A person can be in a physical and a virtual environment at the same time. For example, this person could be at his work office and at the same time participating in a video-conference with a team located in another continent. He may have different roles in each of these two environments. The model is able to capture these different aspects of the user context both in the physical and the virtual world. Figure 7 shows a detailed view of the environment element.

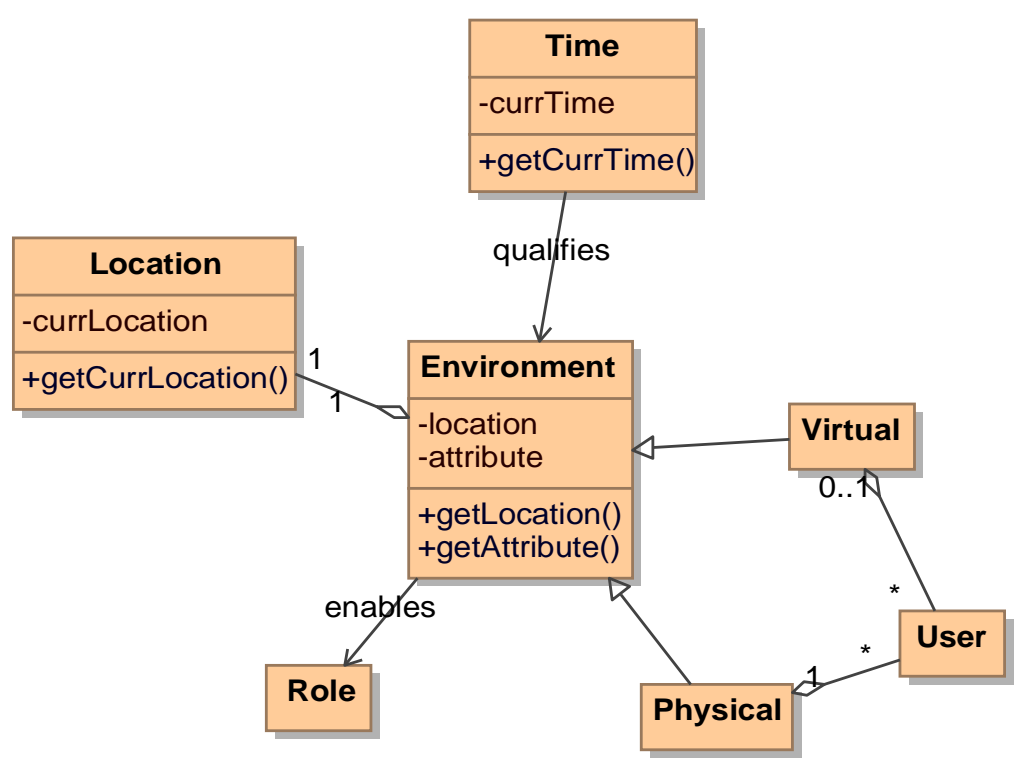

Figure 7 Environment view

\subsection{Dynamic Behaviour}

A dynamic interaction between context elements can be specified through sequence diagrams. Figure 8 shows the interactions among context elements: task, profile, role and preference. This sequence diagram illustrates the dynamic changes triggered by a change in task. This change is propagated to the user profile which updates itself. The profile then notifies the role and the preference elements in 
parallel. Both role and preference may respond to this notification and result in further changes in the user context.

For example, the user just finished with his exam, and he marks it off from his to-do list. The profile is updated to reflect that the task is done and the time needed for studying decreases. The user role changes from a student role to leisure role. The user's preference changes form prioritizing study time to prioritizing entertainment.

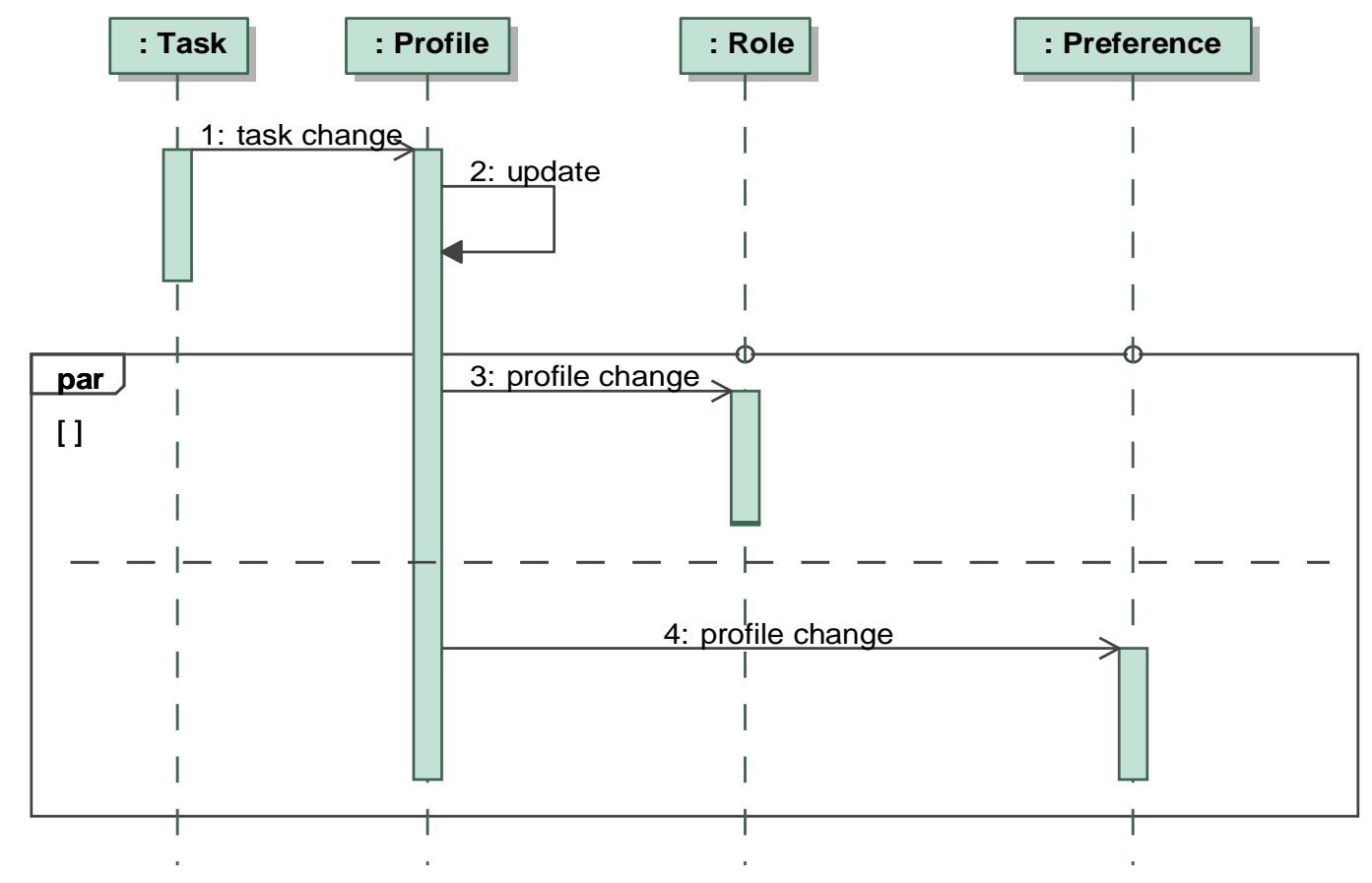

Figure 8 Dynamic interactions between task, profile, role and preference context elements

Figure 9 illustrates dynamic changes triggered by a change in task as well, and it shows the interactions among context elements, task, role and preference. The role and the preference elements are notified in parallel regarding the change in task and may update themselves. The role change may result in additional changes in the preference element. 


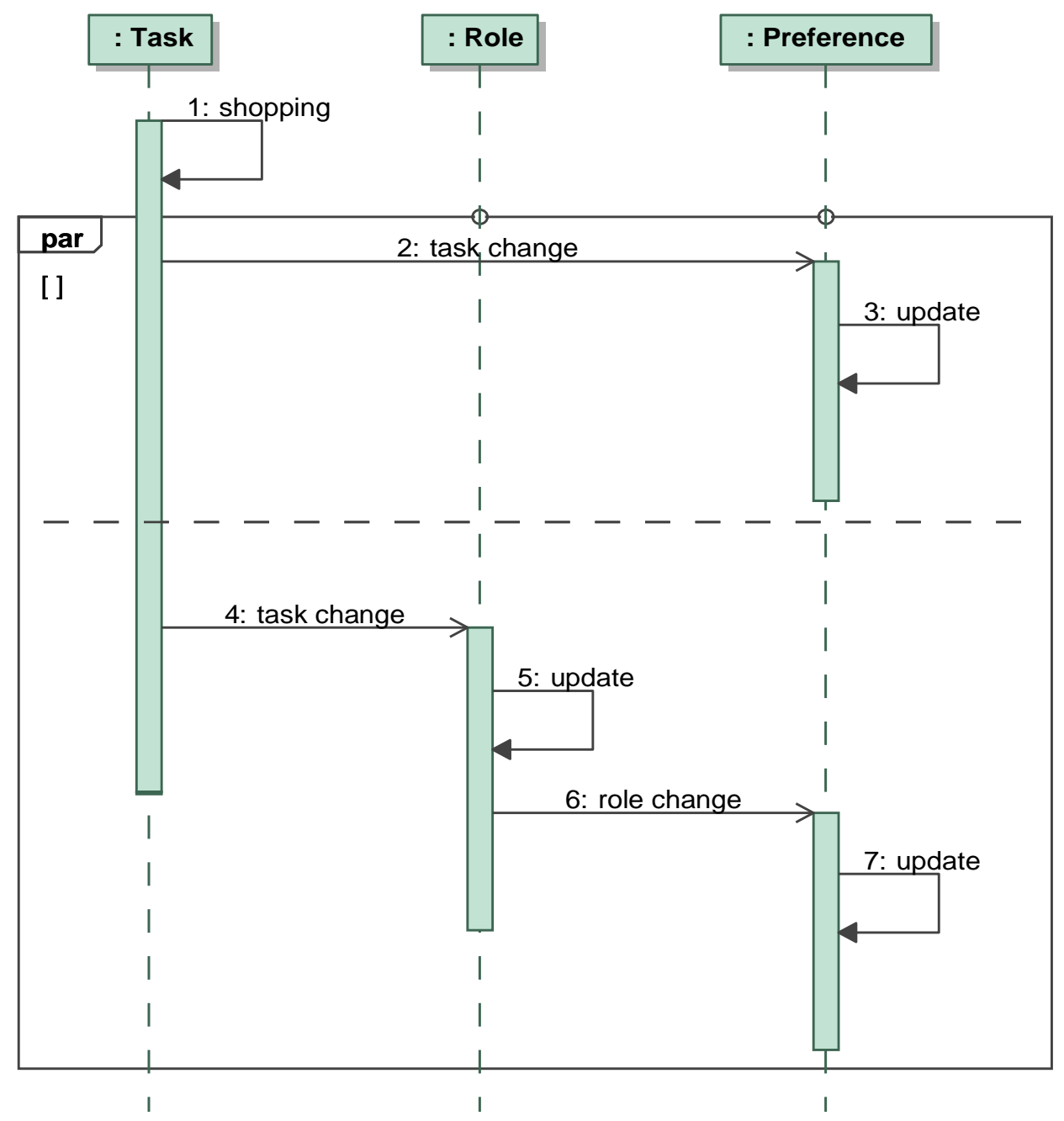

Figure 9 Dynamic interactions between task, role and preference context elements

There are also dynamic interactions between environments and roles as well as groups and preferences. The changes in the physical or virtual environment may enable new roles. This role adaptation may propagate to the preference element. The changes in the social relationships of the group may influence user preference as well.

\subsection{Conclusion}

This chapter has presented the design of the dynamic user-centric context model. The model captures the dynamic interactions involving user context and virtual environments. The design of the context 
model is illustrated through the class diagrams. The overall design is in Figure 1 and specific context element views are from Figure 2 to Figure 7. The context model captures roles, preferences, and social relationships, which are essential to capturing user perspectives in a user-centric model. The model also incorporates virtual environments to model online interactions, and it considers other contextual elements such as tasks and profiles. The potential dynamic interactions are illustrated through the sequence diagrams in Figure 8 and Figure 9. 


\section{Chapter 5}

\section{Case Studies}

This chapter presents four case studies related to the following four domains: mobile e-healthcare, mobile commerce, mobile tourism, and mobile augmented reality gaming. The first case study illustrates a scenario where a context-aware mobile device assists a nurse in her routine work. The second case illustrates a context-aware mobile device providing personalized information and services while the user shops. The third case outlines a scenario where the mobile device becomes a personalized tour guide. The last case study shows a mobile augmented reality game that can be deployed in a museum.

Each case study focuses and showcases a particular aspect of the model. The first case study on mobile e-healthcare shows the dynamic interactions between the role context and other contextual elements. The second case study on mobile commerce shows the adaptation of the preference context triggered by changes in other contextual elements. The third case study on mobile tourism shows the impact of different types of social relationships on the preferences context. The last case study on mobile augmented reality gaming shows the dynamic interactions between contextual elements in virtual environments. Table 5 summarizes the dynamic interactions between contextual elements in each scenario. The numbers in the columns represent the case studies that model the particular interaction. The dynamic interactions involve tasks, preferences, roles, groups, and physical and virtual environments.

The case studies apply the context model to demonstrate the sample usage scenario of the model. These scenarios showcase the potential applications and functionality that can be instantiated from the model. The following sections discuss the details of each case study by presenting the scenario used and the dynamic context interactions shown by each. 


\begin{tabular}{|c|c|c|}
\hline \multicolumn{2}{|c|}{ Dynamic Interactions } & Case Study \\
\hline Cause: changes in & Effect: changes in & 1,2 \\
\hline Task & Role & 2,4 \\
\hline Task & Preference & $1,2,4$ \\
\hline Role & Preference & $2,3,4$ \\
\hline Group & Preference & 1,2 \\
\hline Physical Environment & Role & 1,4 \\
\hline Virtual Environment & Role & \\
\hline
\end{tabular}

Table 5 Summary of dynamic interactions shown by each case study

\subsection{Mobile E-Healthcare}

This case study extends the scenario described in [34] to include preferences and social relationships other than location, time and role. This case study assumes Angela is a nurse working at a hospital. Her schedule consists of mostly morning and afternoon shifts, with occasional evening shifts.

\subsubsection{Scenario}

As Angela approaches the hospital building, close to her shift time, her mobile device detects that the hospital network is within range and connects to it. Even though she is not physically in front of her desk, she now has partial access to her work and receives notifications for her work today through this virtual environment.

Once she enters the hospital, the mobile device detects her presence in this physical environment. It changes her role and gives her full access to patient files and other work related data. This change in role affects her mobile device which now operates in the professional mode. This profile configures the mobile device to vibrate mode and displays her work related task list.

Angela starts her routine checks on patients. Her mobile detects her current task and updates her role to be checkup nurse. Her preferences change accordingly, with the mobile device configured to display a brief profile of the next patient and the remaining time she has for the current patient based on number of patients and available time left. 
As she enters a patient's room, her mobile device communicates with the hospital network and is notified that Angela is qualified to be an extra duty nurse. Angela is the first nurse checking up the patient after Dr. Smith's visit. Dr. Smith has instructed the next nurse to take the blood sample of the patient for a blood test. Angela's mobile device updates her role to be extra duty nurse, and her task list is updated with a new task set by Dr. Smith. After Angela finishes taking the blood sample of the patient, her mobile device reminds her that the next task is to give the patient medicines. Her device also gives her the patient's medical record in the system so she can detect any inconsistencies between the record in the system and medicines she actually has on her cart. At the end, Angela logs her actions and observations on the patient. Dr. Smith will automatically receive the log, and the log will be accessible to other doctors and checkup nurses.

\subsubsection{Dynamic interactions between contextual elements}

As shown in Table 5, this first case study shows the dynamic interactions initiated by task, physical environment, and virtual environment contexts. These changes trigger updates in the role context, and the changes propagate to the preference context.

\section{Virtual and physical environments initiated interactions with roles and preferences}

The virtual environment component receives a notification from the Wi-Fi module, and the hospital network is available to Angela based on her current location and time. The Wi-Fi module connects to the hospital network and notifies the virtual environment component which updates itself to

HospitalNetworkVirtualEnvironment. This change in the virtual environment propagates to the role component. The role component updates the dominant role to VirtualNurse.

The physical environment component receives Angela's location from the location sensors, and Angela is in her office in the hospital. It verifies that Angela is located within her office boundaries in the hospital. The physical environment component also checks the current time and Angela's shift time. It changes to WorkEnvironment and notifies the role component. The role component changes the dominant role to Nurse and propagates this change to the preference component. The preference component adapts to the new Nurse role by setting the dominant set of preferences to

ProfessionalMode. The volume of the device is muted and vibrate mode is on. Information related to the work of the day is provided. 
The physical environment receives an update on Angela's location, and it verifies that Angela is within the patient's room boundaries. Angela is the first checkup nurse after the doctor's visit. The physical environment component updates itself to SpecialInstructionAvailableEnvironment. This change propagates to the role component which changes the dominant role to ExtraDutyNurse. This update then triggers another notification which is sent to the preference component. It updates the dominant set of preferences to ExtraDutyNurseHelp based on the previously defined rules to facilitate the new ExtraDutyNurse role. For example, automate actions, such as update the task list and notify the doctor that the task is completed, are triggered.

The object diagram shown in Figure 10 demonstrates a partial instantiation of the context model to describe the role and preference adaptations initially triggered by the virtual and the physical environments changes. Figure 11 shows the sequence of these dynamic interactions described in this mobile e-healthcare scenario.

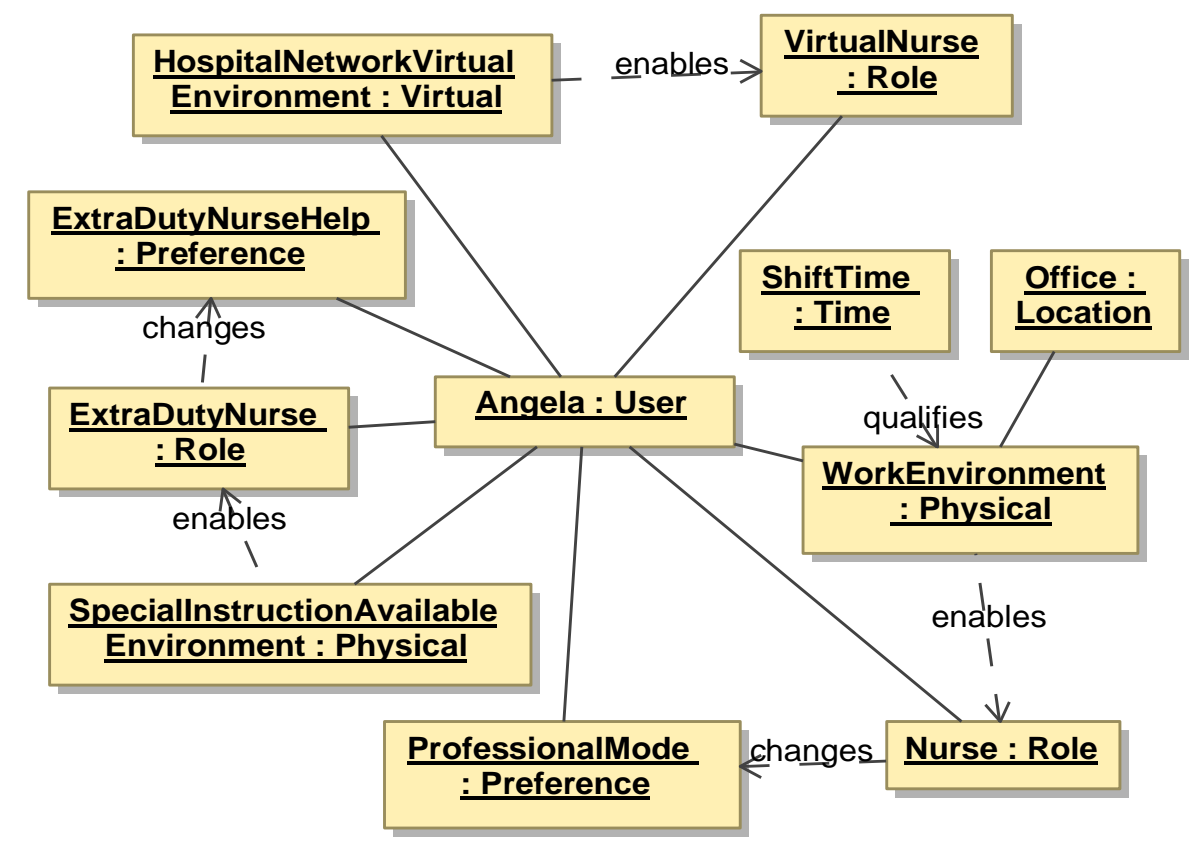

Figure 10 Object diagram describing changes triggered by environments changes in the mobile e-healthcare scenario 


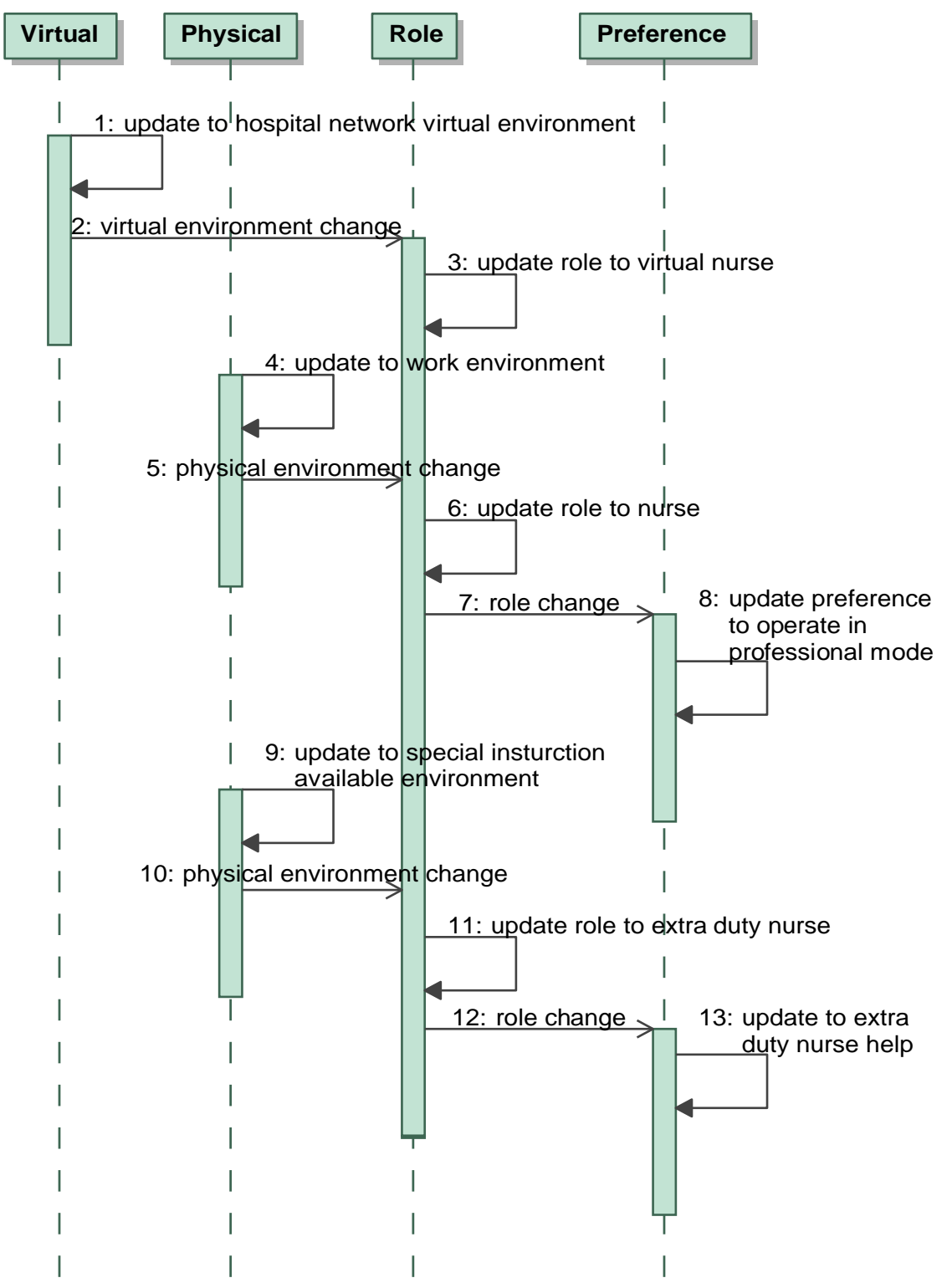

Figure 11 Sequence diagram describing changes triggered by environment changes in the mobile e-healthcare scenario

\section{Tasks initiated interactions with roles and preferences}

The task component determines that Angela is currently checking up patients based on her calendar and her current location. It updates the current task to be CheckingUpPatients. This change propagates to the role component, and the role component updates itself to CheckupNurse. This 
change triggers a notification that is sent to the preference component. The preference component updates itself to CheckupNurseHelp to facilitate the tasks that a checkup nurse has to perform. In this case, the user interface displays specific information, such as patient information and time left for this patient.

Figure 12 demonstrates a partial instantiation of the context model to describe the role and preference adaptations triggered by task change. Figure 13 summarizes the sequence of adaptations triggered by task change described in this mobile e-healthcare scenario.

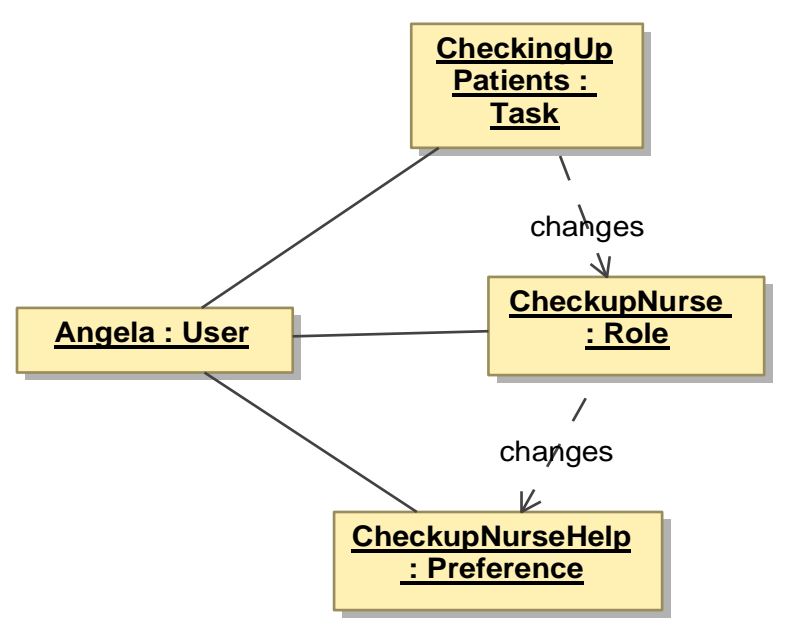

Figure 12 Object diagram describing changes triggered by task change in the mobile ehealthcare scenario 


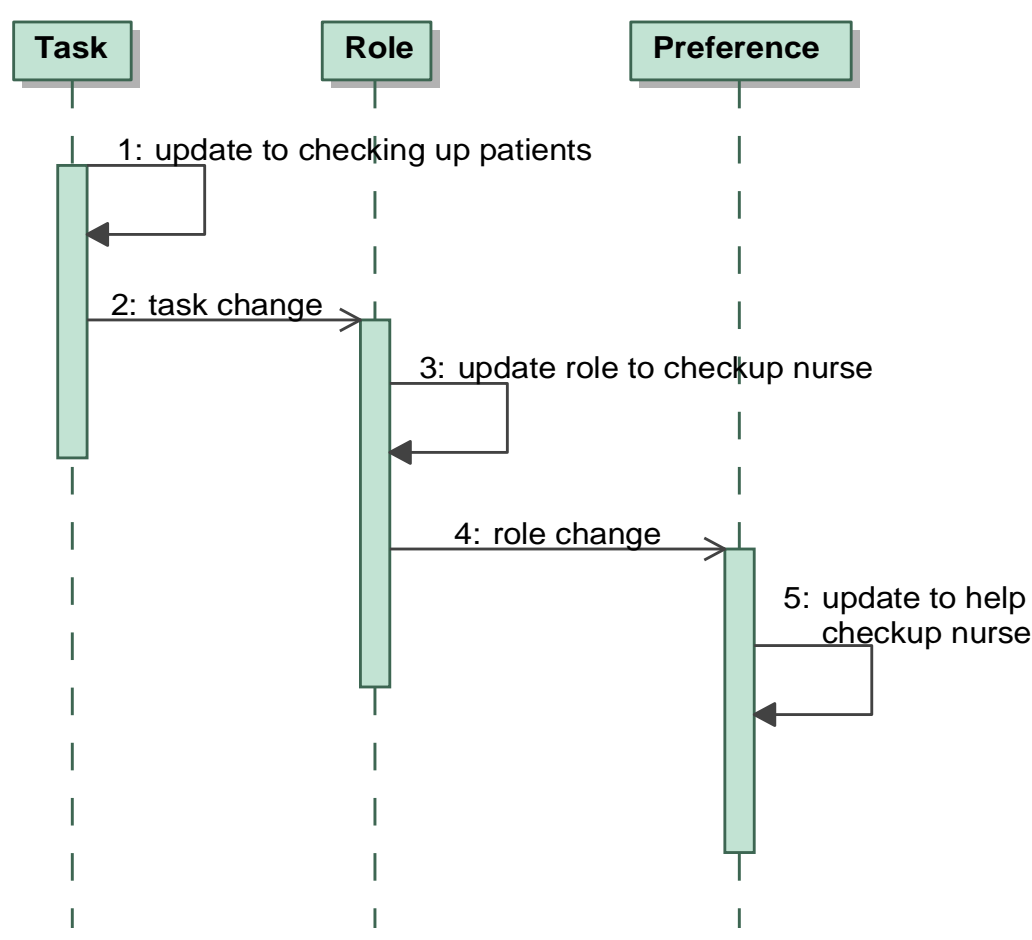

Figure 13 Sequence diagram describing changes triggered by task change in the mobile ehealthcare scenario

\subsection{Mobile Commerce}

Continuing from the first case study in mobile e-healthcare, this case study outlines a scenario which describes Angela as a mother after work. The scenario presents how Angela's mobile device helps her while she shops.

\subsubsection{Scenario}

At the end of Angela's shift time, she exits from the hospital. Her device deactivates her nurse role, and updates her dominant role to mother. The type of advertisements Angela receives changes accordingly. The amount of professional related advertisement is reduced, and the amount of casual role related advertisement is increased. For example, the amount of advertisement related to comfortable nurse shoes or patient care workshop is reduced. Angela now receives more advertisement from grocery stores or clothing retailers. 
Angela searches for dresses that she can wear to her friend's wedding, and her mobile device presents her the search results as well as some related coupons and promotions. Angela browses through the coupons, and the device updates her interest profile to reflect the new item of interest. Later, the device can notify Angela about items on sale or new arrival items that she will potentially be interested based on her interest profile.

Angela is going to meet up with her friend, Melody, to shop for dresses together. On her way to the mall, Angela's mobile device turns the volume to the max since she is taking the bus. Her mobile device also adjusts the font size so it is easier to read on the bus.

As Angela enters the mall and starts to search for a dress, the mobile device sets Angela's role to shopper. It shows her the mall map and highlights potential stores that she would be interested as well as stores that she had viewed the coupons. Once Angela meets up with Melody, the mobile device detects Melody's presence and it prompts Angela if she wants to connect their devices via Bluetooth. Angela chooses to connect with Melody's device. Once the connection is established, the device suggests coupons and stores in which both of them would be interested.

Angela and Melody enter the store highlighted as a store in which Angela would be interested. Angela picks up a dress and turns on the function of adding to shopping list for comparison. The mobile device connects with the store network, and it is able to provide Angela the price and extra information such as fabric used and washing instructions related to the dress.

After Angela finishes her shopping at the mall, she drops by a grocery store on her way home. Her mobile connects with the store network and loads Angela's shopping list. The promotion items are highlighted on the shopping list. Her mobile device shows a map of the grocery store, and points out where the item is in the store. After the mobile device establishes a connection with the grocery store's network, Angela's role is updated to virtual grocery shopper. Angela turns on the comparison button on her device, and the items that are also on promotion in other grocery stores are underlined. The mobile device considered the grocery stores that Angela visits the most frequently.

Angela just arrived home, and her mobile device updates her interest profile based on the items she actually bought today. Her interest profile can be used to provide better shopping suggestions in the future. The items Angela actually bought would have higher precedence in the interest profile. 


\subsubsection{Dynamic interactions between contextual elements}

This mobile commerce scenario shows the adaptations of preferences triggered by changes in roles, the group around, and tasks as shown in Table 5.

\section{Preference adaptations to role change involving environments}

The physical environment component receives an update from the location sensors, and Angela has left the hospital. It verifies that Angela is not within her office boundaries, and it also checks the current time and Angela's shift time. It changes to AfterWorkEnvironment and notifies the role component. The role component updates the dominant role to Mother and propagates this change to the preference component. The preference component updates to LeisureMode which prioritizes different things to facilitate the Mother role. Work related events are not the top priority now. For example, the new advertisement preference setting would decrease the amount of work related workshop advertisements and increase the amount of promotions and coupons for grocery.

The object diagram, as shown in Figure 14, demonstrates a partial instantiation of the context model to describe the preference adaptations triggered by role change involving the physical environment context. Figure 15 summarizes the sequence of preference adaptations described in this mobile commerce scenario.

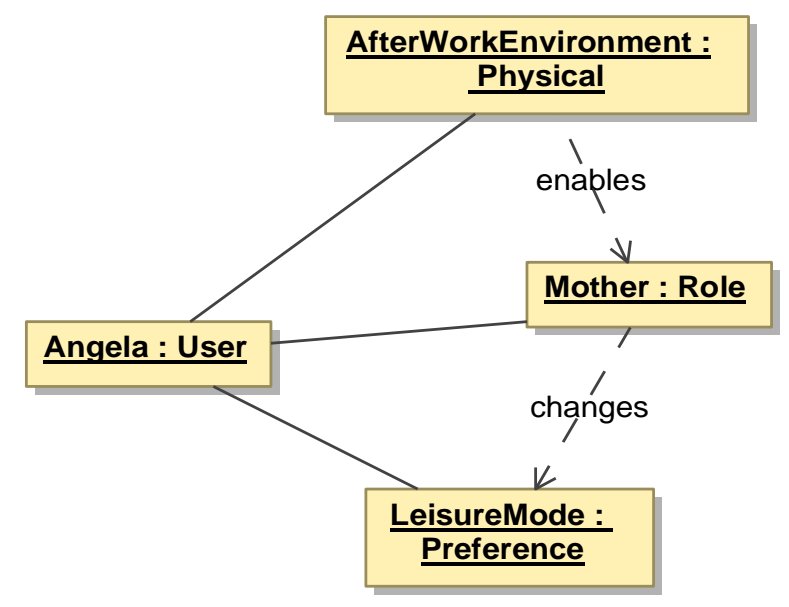

Figure 14 Object diagram describing preference adaptations triggered by role change in the mobile commerce scenario 


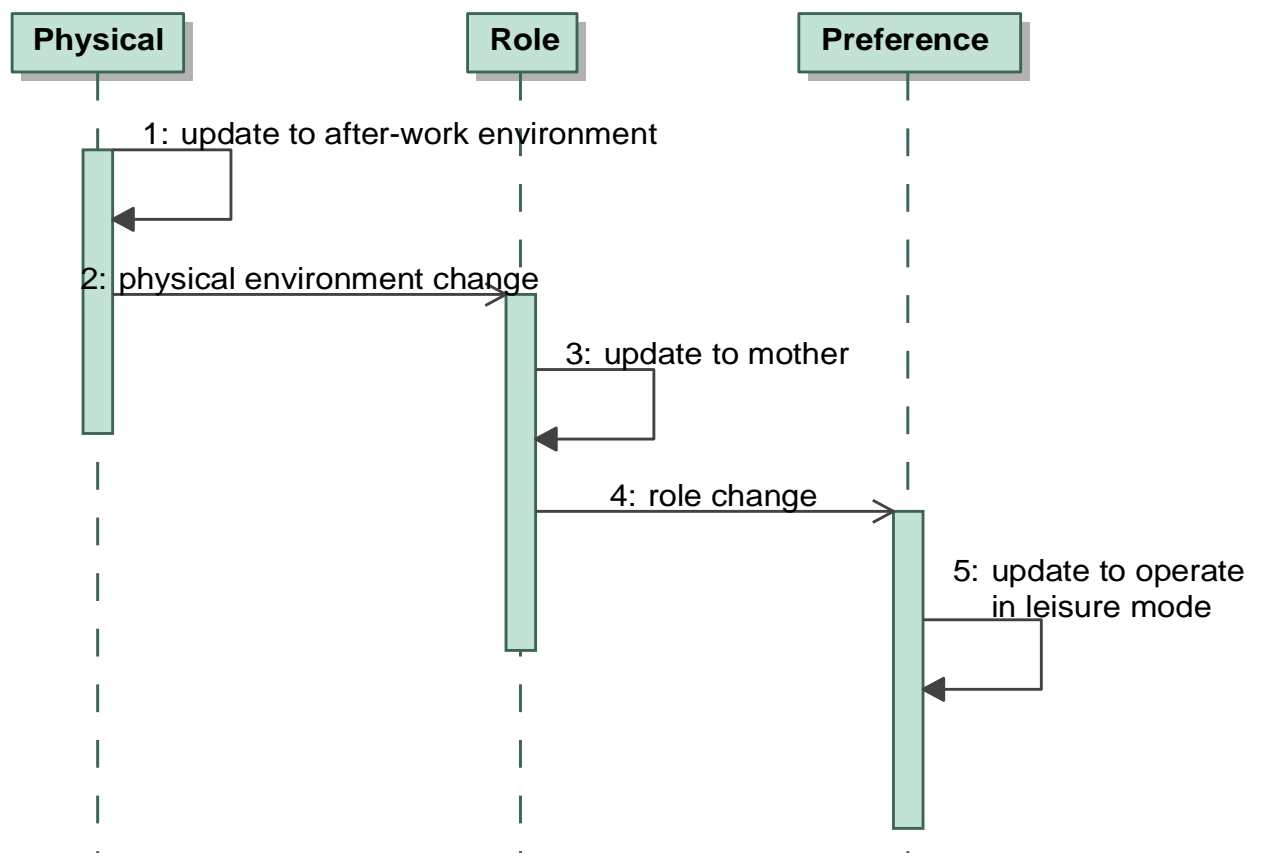

Figure 15 Sequence diagram describing preference adaptation triggered by role change in the mobile commerce scenario

\section{Preference adaptations to role and task changes}

The task component determines that Angela is currently shopping for a dress based on her calendar and current location. It updates to Shopping and notifies the role component which changes the dominant role to Shopper. This change propagates to the preference component. The preference component updates to GeneralShoppingHelp to retrieve the preference settings relevant to the Shopper role and prioritize them. The promotions, coupons, and store locations in mall were set by Angela to be the most relevant information in this situation.

The task component receives a request from Angela to provide information on a dress, and it updates itself to CheckingItemInformation. It notifies the preference component which updates itself to CheckInformationHelp. The preference component retrieves the preference settings related to checking information on a dress and prioritizes them. The price and washing instructions have been customized to be the most interested information in this situation.

The object diagram, as shown in Figure 16, demonstrates a partial instantiation of the context model to describe the preference adaptations triggered by role and task changes. Figure 17 
summarizes the sequence of preference adaptations triggered by role and task changes described in this mobile commerce scenario.

\section{Preference adaptations to group change}

The group component receives an update on the user around Angela from the Bluetooth module. A user, Melody, in Angela's social networks, is in proximity. The group component checks the calendar and is notified that Angela and Melody are planning on shopping together. With proper permission, the Bluetooth module establishes a connection with Melody's device and notifies the group component which updates to FriendAround. The group component notifies the preference component which changes to ShopTogetherMode. As previously set by Angela, the preference component incorporates Melody's previous bought items to form Angela's new shopping preferences.

The object diagram, as shown in Figure 18, demonstrates a partial instantiation of the context model to describe the dynamic interactions between preferences and groups. Figure 19 summarizes the sequence of dynamic interactions between preferences and groups in this mobile commerce scenario.

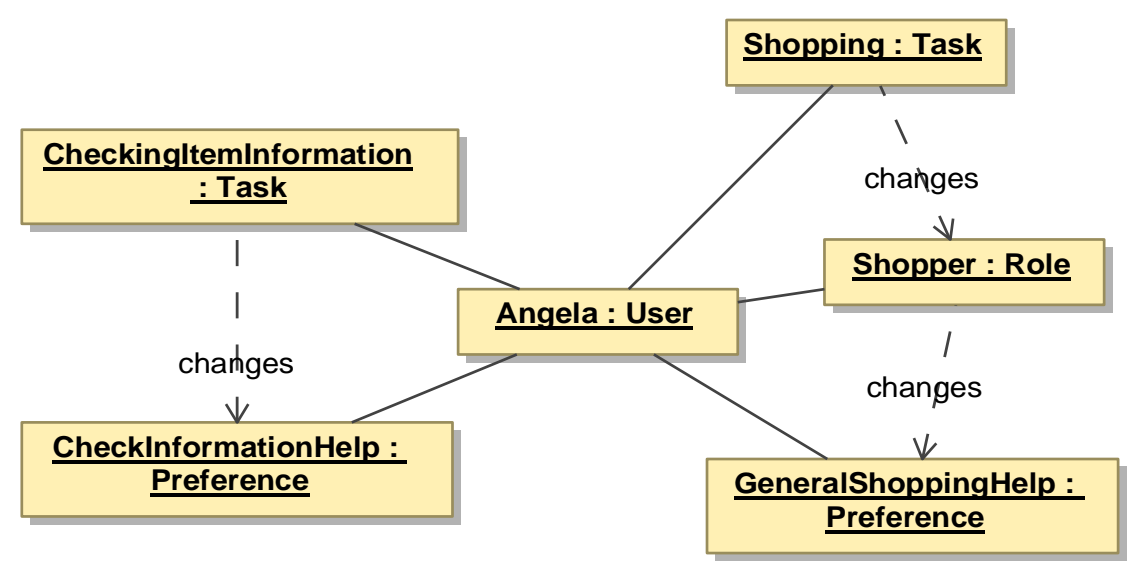

Figure 16 Object diagram describing preference adaptations triggered by role and task changes in the mobile commerce scenario 


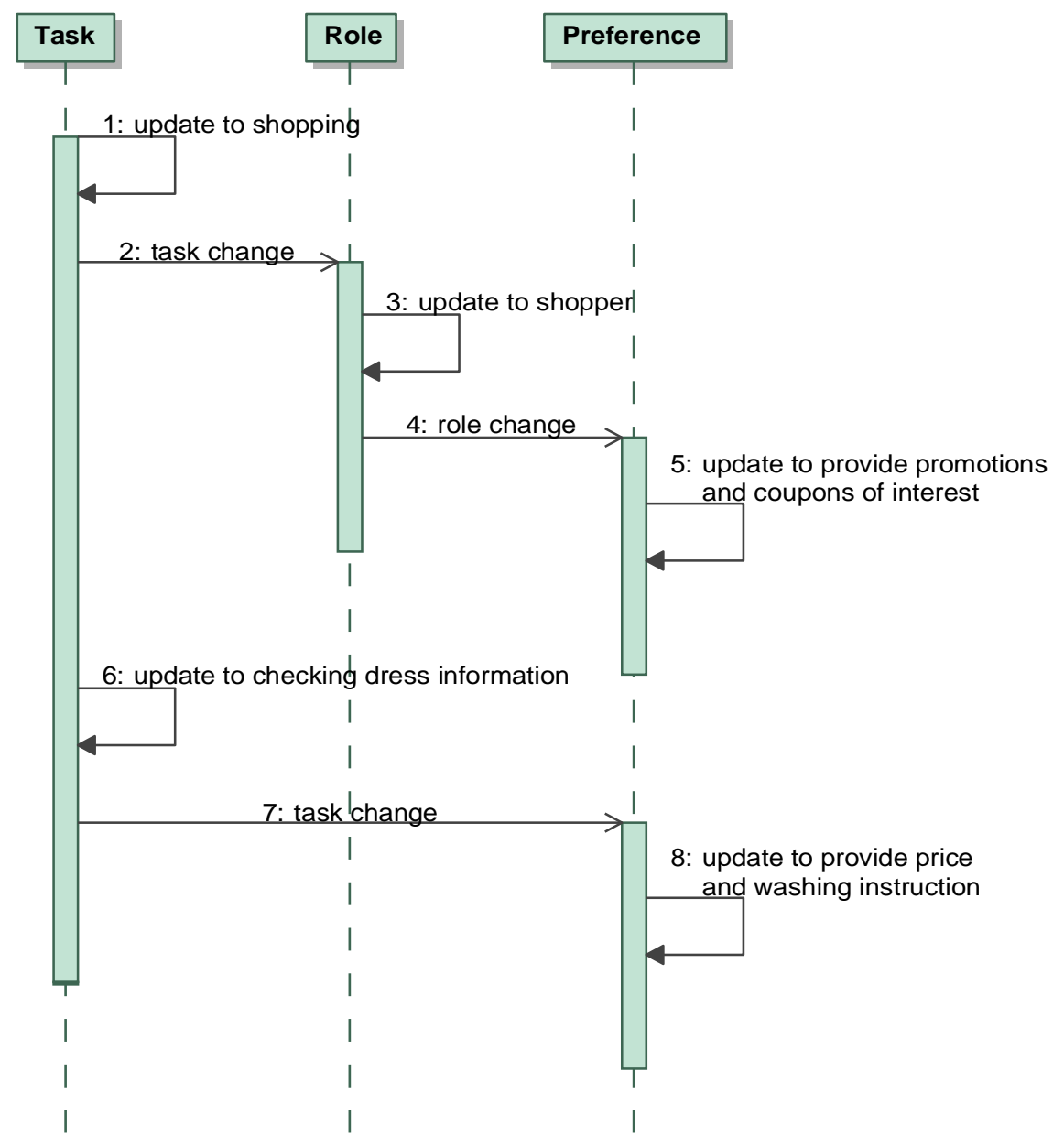

Figure 17 Sequence diagram describing preference adaptations triggered by role and task changes in the mobile commerce scenario 


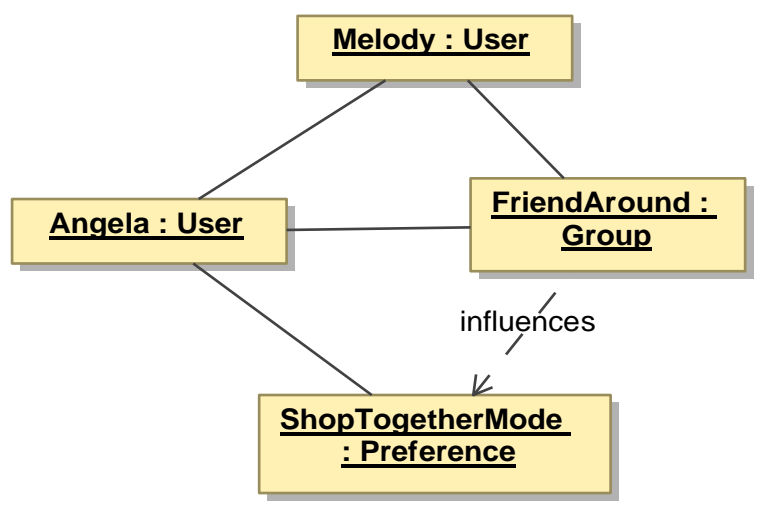

Figure 18 Object diagram describing the dynamic interactions between preferences and groups in the mobile commerce scenario

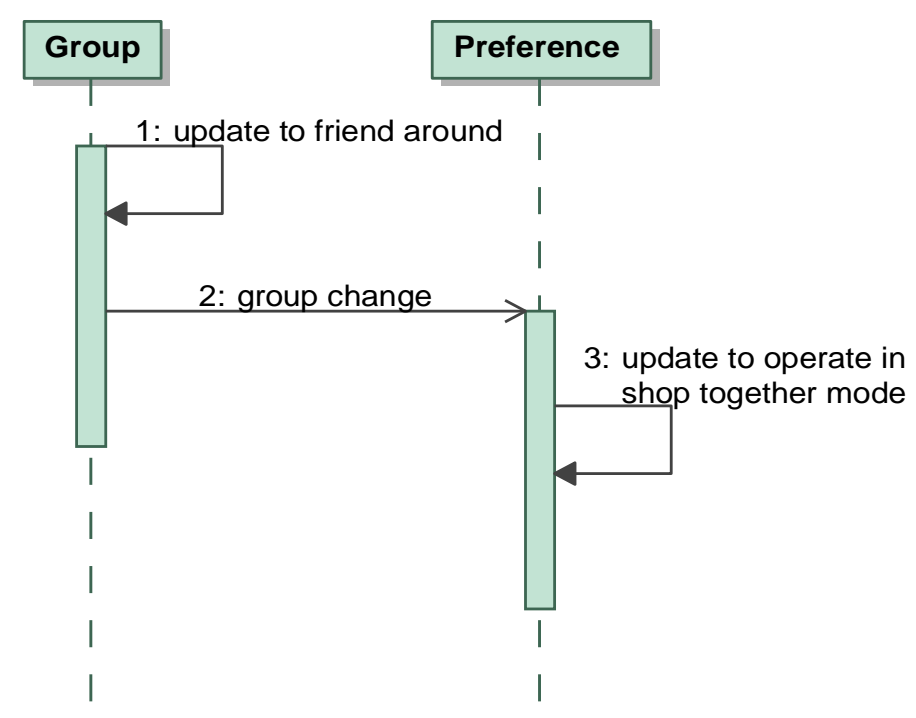

Figure 19 Sequence diagram describing dynamic interactions between preferences and groups in the mobile commerce scenario

\subsection{Mobile Tourism}

Continuing from the first two case studies in mobile e-healthcare and mobile tourism, Angela is going to a conference and then taking a vacation in Milan, Italy. The scenario presents how Angela's mobile device helps her while she travels. 


\subsubsection{Scenario}

Angela sets her device to vacation mode. Her mobile device would notify anyone who calls her that Angela is on vacation now, and suggest that the caller choose to stay on the line or hang up and contact her through other means.

Angela has arrived in Milan with her colleagues for a conference on patient care. During lunch, the mobile device displays names of people sitting at the same table and their professional information. It helps Angela to remember names of others. It also shows Angela their association with the conferences. For example, the person sitting next to Angela may be in the conference committee or will be presenting a talk.

The next day, Angela starts her vacation in Milan. In order to decide her trip for the day, Angela browses through the recommendations made by her mobile device. Angela's device makes suggestions based on the popular attraction sites in the area, ratings of tour sites, and suggestions from people in Angela's social networks. Angela selects a few attractions to add to her agenda, and her device updates her tourist profile. Her device plans a personalized trip for her based on her selection, her interest profile, and the attributes of the attraction sites. For example, the operation hours of attraction sites and weather condition are taken into considerations.

As Angela starts her tour, her mobile device becomes a personal tour guide. Since Angela has not arrived at her destination, her device sets her to be in the travelling mode. The volume of her phone is turned to maximum, and the map and instructions for getting to the attraction site are displayed on her phone. Her role is set to traveller, and her device focuses on helping Angela reach the destination. It does not try to explain the history of the transportation system in Milan or provide information that is not relevant to reaching the destination.

Angela arrives at the destination, a cathedral, and the mobile device updates Angela's role to visitor. The visitor role sets the mobile device to focus on providing information on the attraction site. Angela receives information such as some must-sees in the attraction site recommended by other visitors as well as the information on the tour groups led by cathedral staff.

Angela chooses to join the cathedral tour group, and her device adds other tour group members to her tourism network. The tour guide shows them the famous attractions in the west wing of the cathedral, and then all the group members have half hour to explore the rest. Angela and her new 
friend, Sharon, from the tour group decide to explore the cathedral together, and they connect their devices together through Bluetooth. The device considers Angela and Sharon's background and preferences to make recommendations. They are more interested in the archaeological and cultural aspects of the cathedral.

Angela updates the recommendation criteria to include comments made on the cathedral. Her device first searches through Angela's social networks to check if her friends have made comments on the cathedral. The comments are presented to Angela for her to decide if she would like to see a particular part of the cathedral.

Angela's device also keeps track of the comments made by others from the tour group. It notifies Angela when other members have made new comments which record their new findings. One of the tour group members, Mike, just made a comment on a fresco. Mike says "Come check out this fresco! It's so cool! " The device displays the comment and provides the directions to the commented art and the preview of the art. Angela and Sharon decide to go over and check out the fresco Mike is talking about.

As Angela stands beneath the fresco, she chooses to see the comments made by others. The mobile device connects with the cathedral network. Once her device is connected with the network, it updates her role to virtual visitor. She now has access to the previous comments made by other visitors, and her mobile device presents her all the relevant comments on the fresco.

\subsubsection{Dynamic interactions between contextual elements}

This mobile tourism scenario shows the impact of different types of social relationships on the preference context, as recorded in Table 5. It provides a personalized visiting experience by incorporating the impact of different types of social relationships.

\section{Work related groups and preferences}

The preference component adapts to changes in roles, tasks and groups. The role component receives a notification from the physical environment component, and the user is currently in the ConferenceEnvironment. The role component updates the dominant role to ConferenceAttendee and notifies the preference component about this change. During lunch time, the task component updates itself to HavingLunch and notifies the preference component. The group component receives a notification from the Bluetooth module, and a group of users characterized by the work related 
social relationship is around Angela. The group component updates itself to

WorkRelatedConferenceAttendeesAround and notifies the preference component. Based on the updates from role, task and group components, the preference component updates itself to

AttendeeHelp which facilitates the lunch with work related attendees in the conference. The most relevant information and help provided are establishing new contacts.

The object diagram, as shown in Figure 20, demonstrates a partial instantiation of the context model to describe the impact of work-related social relationships on the preference context. Figure 21 summarizes the sequence of interaction between work-related group and preferences described in this mobile tourism scenario.

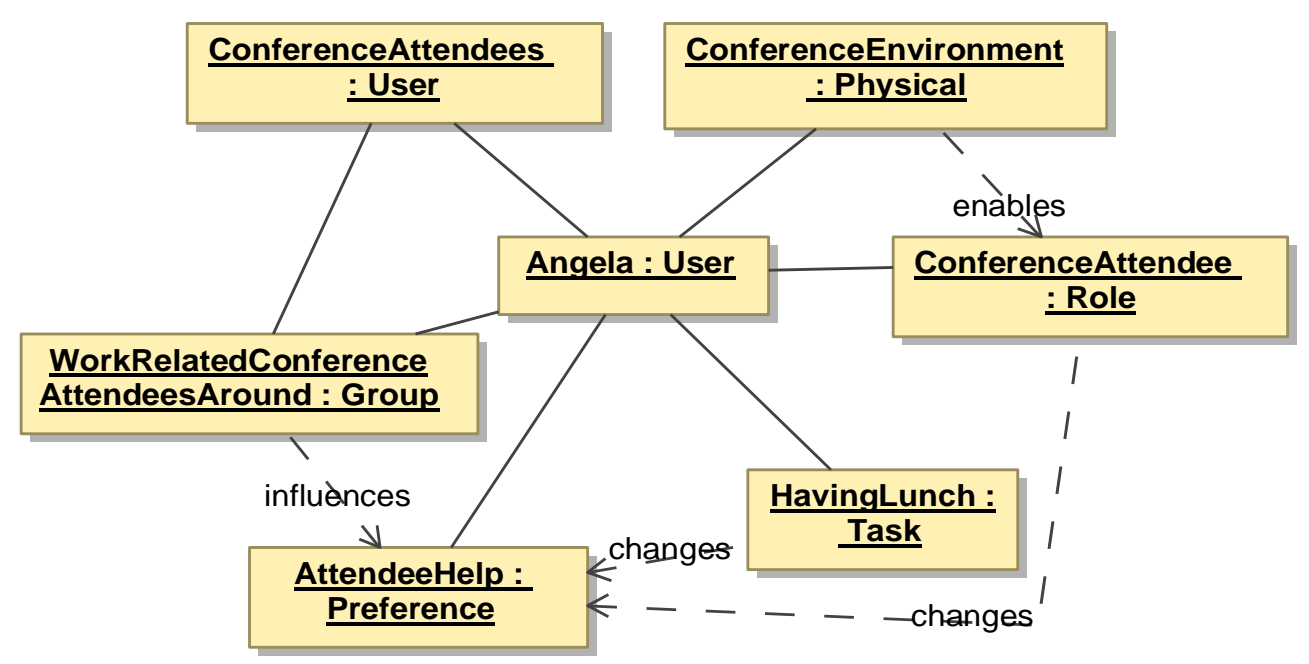

Figure 20 Object diagram describing the impact of work-related social relationships on the preference context in the mobile tourism scenario 


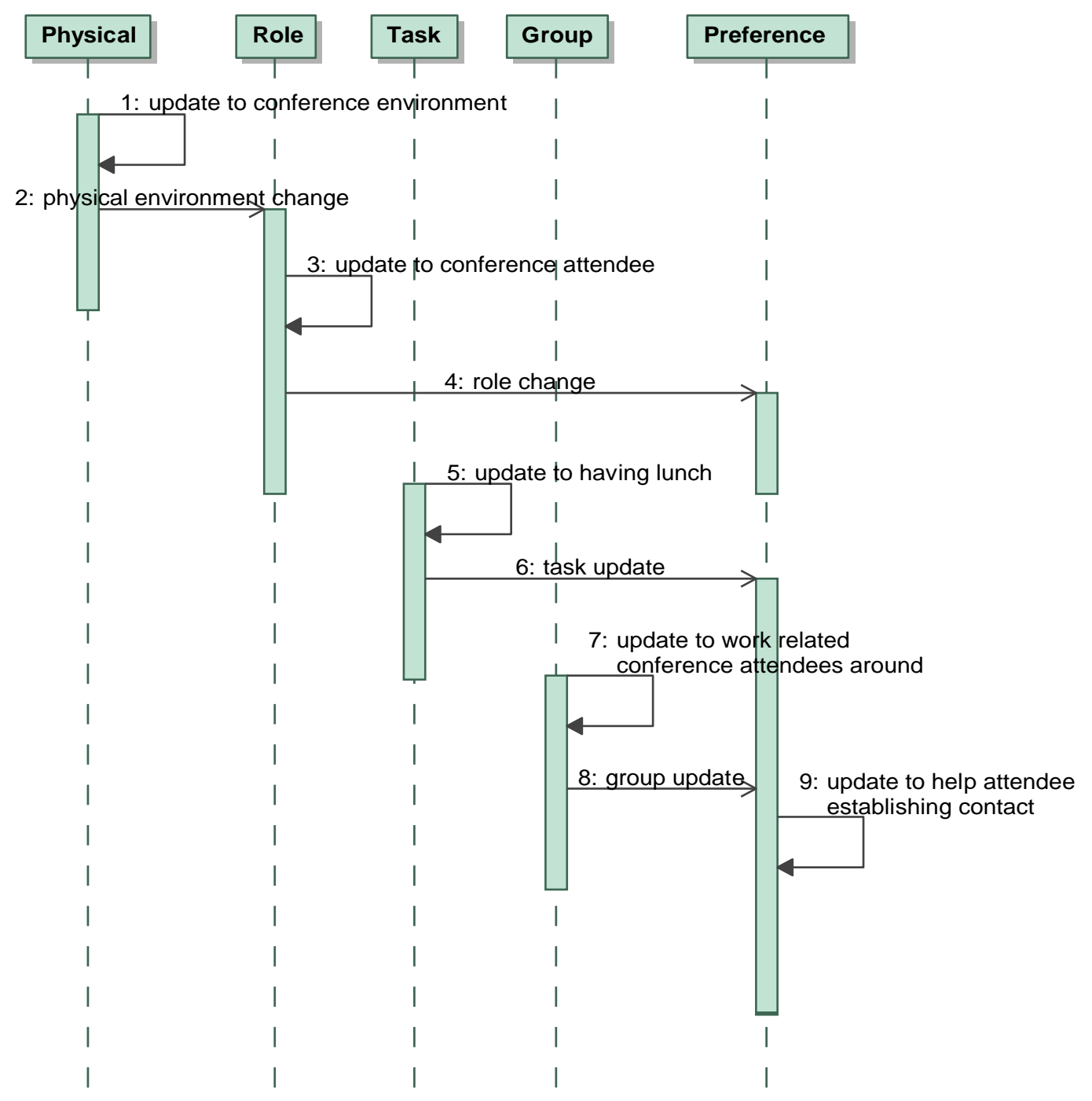

Figure 21 Sequence diagram describing interaction between work-related group and preferences in the mobile tourism scenario

\section{People in the social networks and preferences}

The group component has received the notification that Angela sets her device to be in the vacation mode. The group component sets the friends on Angela's social networks as the default group that would influence the preference component. While planning the personalized trip for Angela, the preference component incorporates the comments made by Angela's friends in her social networks. 


\section{Tour partner, tour group members and preferences}

The group component receives an update from the Bluetooth module, and Angela is connected with another user, Sharon. The group component updates itself to TourPartnerAround based on the social relationship between Angela and Sharon. This change influences the preference component which updates the dominant set of preferences to TourTogetherMode to accommodate the new partner so they both enjoy the tour. Based on Angela's preference settings, the preference component considers Sharon's background and preferences to provide tour recommendations.

The group component monitors the comments updated by tour group members. It receives a notification that Mike has made a new comment. It updates to TourGroupMember and notifies the preference component. The preference component updates to OfInterestHelp which retrieves relevant information to assist users to determine whether the commented art is of the user's interest. It provides directions and preview of the art.

The object diagram, as shown in Figure 22, demonstrates a partial instantiation of the context model to describe the impact of tour partner and tour group members on the preference context. Figure 23 summarizes the sequence of interactions between tour partner, tour group members and preferences described in this mobile tourism scenario.

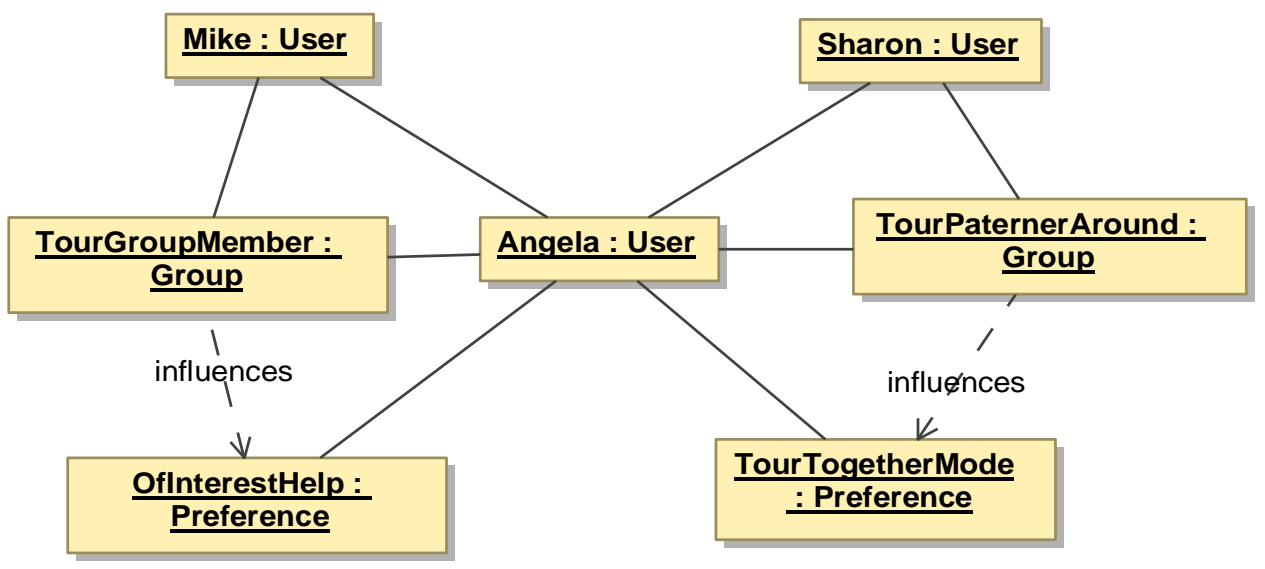

Figure 22 Object diagram describing the impact of tour partner and tour group members on the preference context in the mobile tourism scenario 


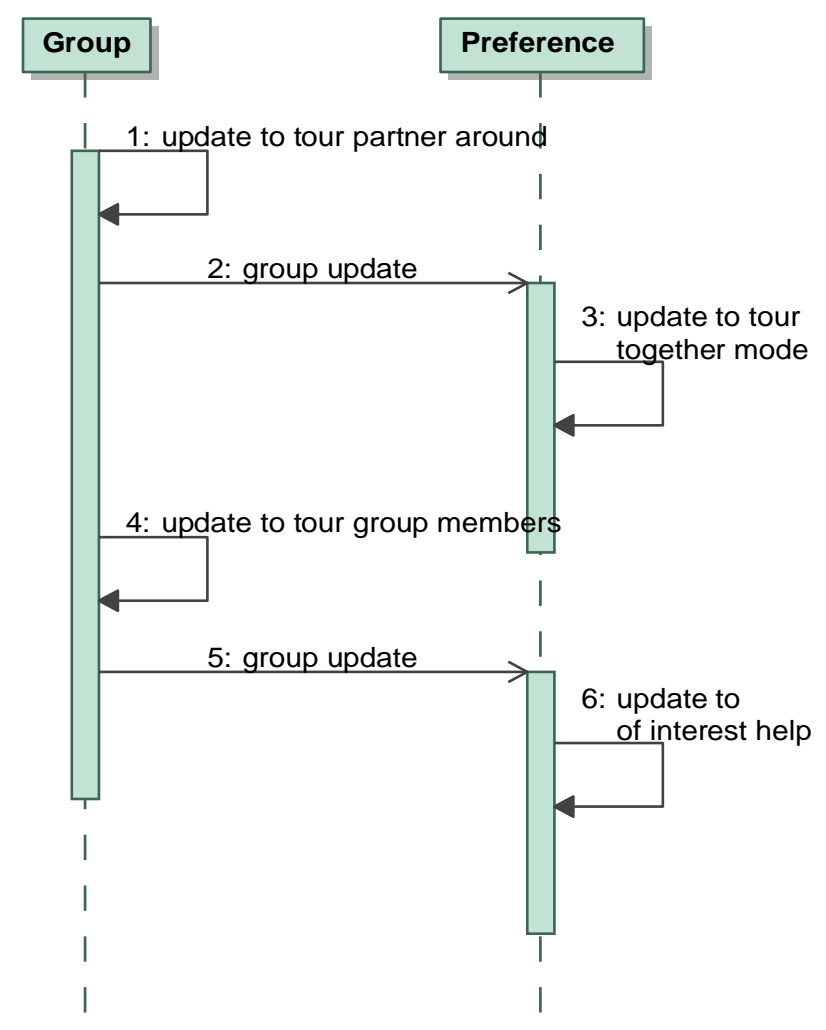

Figure 23 Sequence diagram describing interactions between tour partner, tour group members and preferences in the mobile tourism scenario

\section{Strangers and preferences}

The group component is notified that all the comments regarding the fresco should be displayed, and it updates itself to MuseumVisitors. The preference component receives this update and displays comments from all the current and past museum visitors.

\subsection{Mobile Augmented Reality Gaming}

This last case study presents a mobile augmented reality game to be enjoyed by museum visitors. This scenario presents how the mobile device helps Angela and her family throughout the game. 


\subsubsection{Scenario}

This mobile augmented reality game is designed to provide museum visitors a taste of ancient life. During the game, the mobile device acts as a personal trainer and provides information specific to the environment players are experiencing. The game is designed as a treasure hunt with a storyline. The storyline of the game connects four main activities of ancient living, namely agriculture, hunting, fishing and cooking. The players have to complete the storyline as well as collect the required and bonus items. There will be hints that guide users to complete the storyline, and players need to complete some tasks to receive the hints. Players can form teams to complete the game as well. At the end of the game, the players are scored based on the items collected and the time they finish the game.

Angela and her husband, Sean, along with their child, Nick, decide to participate in the game while they are in the museum. Her mobile device connects with the museum network to download necessary plug-ins to participant in the game. Her child gets a mobile device designed for children to participate in the game.

Angela and Nick form a team, and their mobile devices add each other to the virtual gaming social network. They start the game by going to the initial starting point, and each one of them receives a hint on which station they should be going to through their mobile devices. The device also sets Angela's role to a mobile gamer.

Angela figures out that she should be going to the fishing stage, and her device helps her navigate to the location. Angela has arrived at the correct station. Her mobile device sets her role to fisherman and sends her a list of tasks she has to complete to receive the hint for next stage. The task list Angela receives includes catching fish and selling them to make certain amount of money. The device also sends her specific information on how to complete the tasks.

Angela arrives at the task location. She connects her mobile device to the gaming network, and her device loads the fishing game. She starts to play the game on her device, and her device provides her with related information such as weather forecast, reef map, and suggested location to fish. Since Angela is busy playing the game, her mobile device switches to audio control mode. Angela receives audio output, and she can give commands through voice input. 
Angela successfully avoids all the reefs and arrives at the fishing point. She receives a telescope which gives her the ability to scout her surroundings. Her device records this change to her gaming profile and will notify her when other players are near. When Angela points the camera on her mobile device to another player, the mobile device shows the gaming profile of the other player.

Angela's device notifies her that Sean is around. Since Sean is not in Angela's gaming network, the mobile device switches to encounter mode. Angela can choose to attack, defend or avoid. Each action would have different effects on the other player's game. By choosing attack, Angela may be able to steal some items from Sean or to interfere with Sean's fishing activity. By defending herself, Angela may be able to defend the attack from Sean and to prevent loses. By avoiding Sean, Angela needs to give up her current fishing location and go to somewhere else. Angela decides to defend herself, and she did not lose any fish she caught so far.

On her way to the market, Angela's device notifies her that Nick who is in her gaming network is around, and her device turns to team mode. Angela decides to connect with Nick and share information together.

Angela has finished the goal of the fishing stage, and she receives the hint for the next stage. She moves on to the agriculture stage, and her device updates her role to fruit collector.

\subsubsection{Dynamic interactions between contextual elements}

This mobile augmented reality gaming scenario shows the dynamic interactions between contextual elements in the virtual gaming environment. As shown in Table 5, this scenario illustrates the interactions between tasks, roles, preferences, groups and virtual environments.

\section{Dynamic interactions between virtual environments, roles and preferences}

The task component receives an update that Angela has joined the mobile game. It updates to PlayingMobileGame and sends a notification to the role component. The role component updates itself to MobileGamer.

The virtual environment component receives a notification from the location sensors, and Angela has arrived at the foyer of second floor in the museum. In the physical environment, this information does not provide new meanings other than that Angela is still inside the museum. However, the virtual environment component recognizes this location as the starting point of the fishing stage. It 
changes to FishingStageVirtualEnvironment and notifies the role component which updates to Fisherman. The original MobileGamer role is not replaced but suppressed. This change propagates to the preference component which updates the dominant set of preferences to FishermanHelp to facilitate the Fisherman role. The information considered as relevant includes the updated task list and introductions to the tasks.

The object diagram shown in Figure 24 demonstrates a partial instantiation of the context model to describe the dynamic interactions between virtual environments, roles and preferences. Figure 25 summarizes the sequence of interactions between virtual environments, roles and preferences described in this mobile augmented reality gaming scenario.

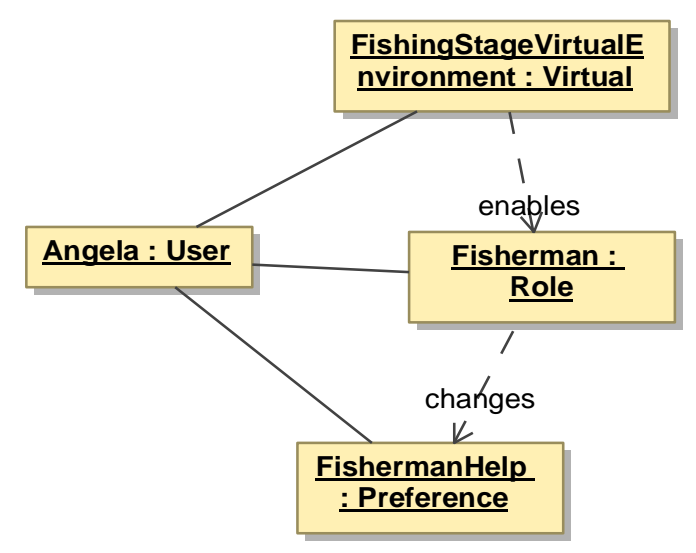

Figure 24 Object diagram describing the dynamic interactions between virtual environments, roles and preferences in the mobile augmented reality gaming scenario 


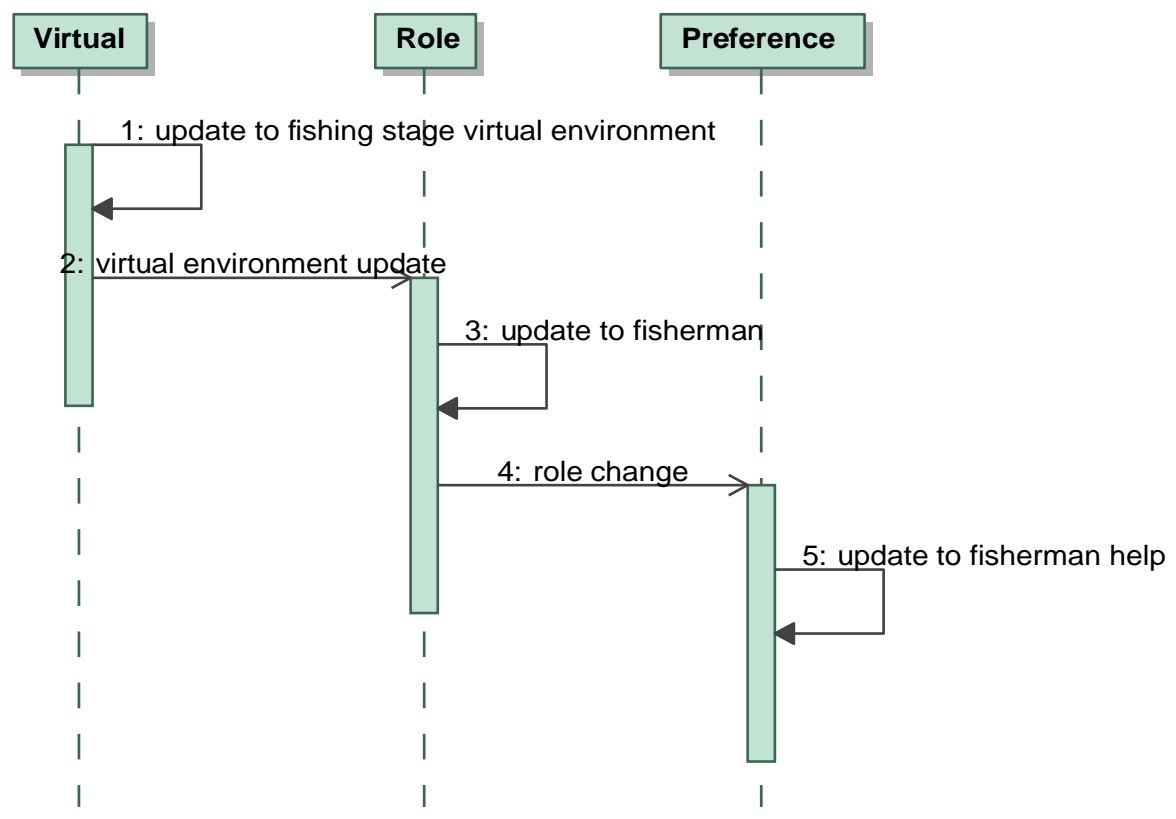

Figure 25 Sequence diagram describing dynamic interactions between virtual environments, roles and preferences in the mobile augmented reality gaming scenario

\section{Dynamic interactions between tasks, groups and preferences in virtual environments}

The task component receives an update from the gaming program, and Angela has started the task on catching fish. It updates to CatchingFish. This change propagates to the preference component. Based on the default preference settings, the preference component updates to FishCatchingHelp and sets the audio control mode to be on. It also displays the information on weather condition, reefs, and suggested location to fish.

The group component receives a notification from the Bluetooth module, and another player who is not in Angela's gaming network is around. Even though this other player, Sean, is Angela's husband in real life, the group component still updates itself to EnemyAround because they are in the virtual environment. This change propagates to the preference component which updates itself to operate in the EncounterMode.

The group component receives an update from the Bluetooth module, and Angela's teammate is around. It updates to TeammateAround. This change propagates to the preference component which updates itself to operate in the TeamMode. Angela and her teammate are connected to share information. 
The object diagram, as shown in Figure 26, demonstrates a partial instantiation of the context model to describe the dynamic interactions between tasks, groups and preferences in the virtual gaming environment. Figure 27 summarizes the sequence of interactions between tasks, groups and preferences in the virtual gaming environment described in this mobile augmented reality gaming scenario.

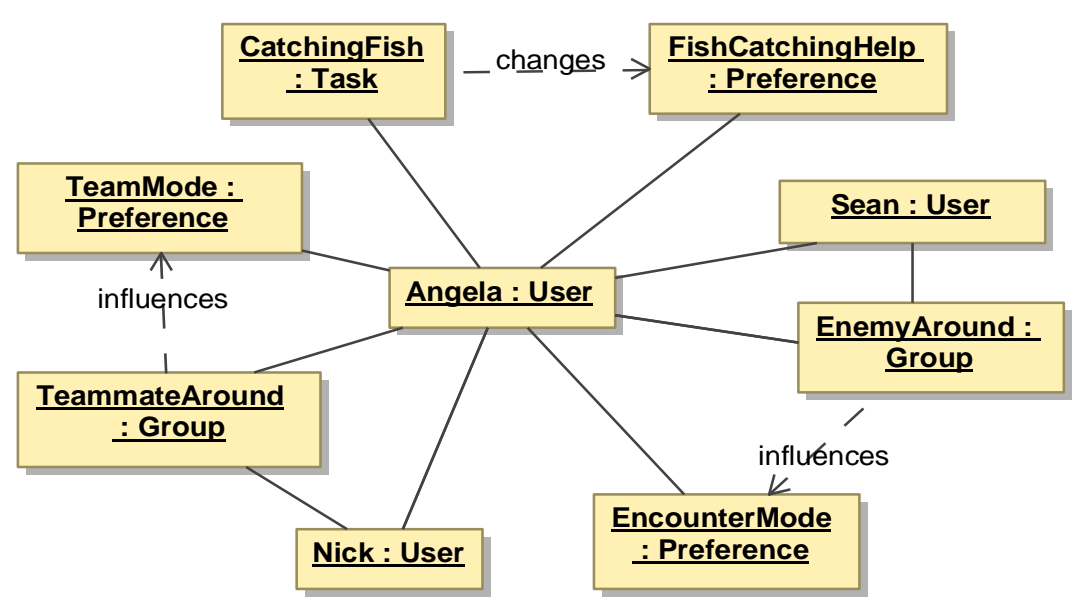

Figure 26 Object diagram describing the dynamic interactions between tasks, groups and preferences in the virtual gaming environment in the mobile augmented reality gaming scenario 


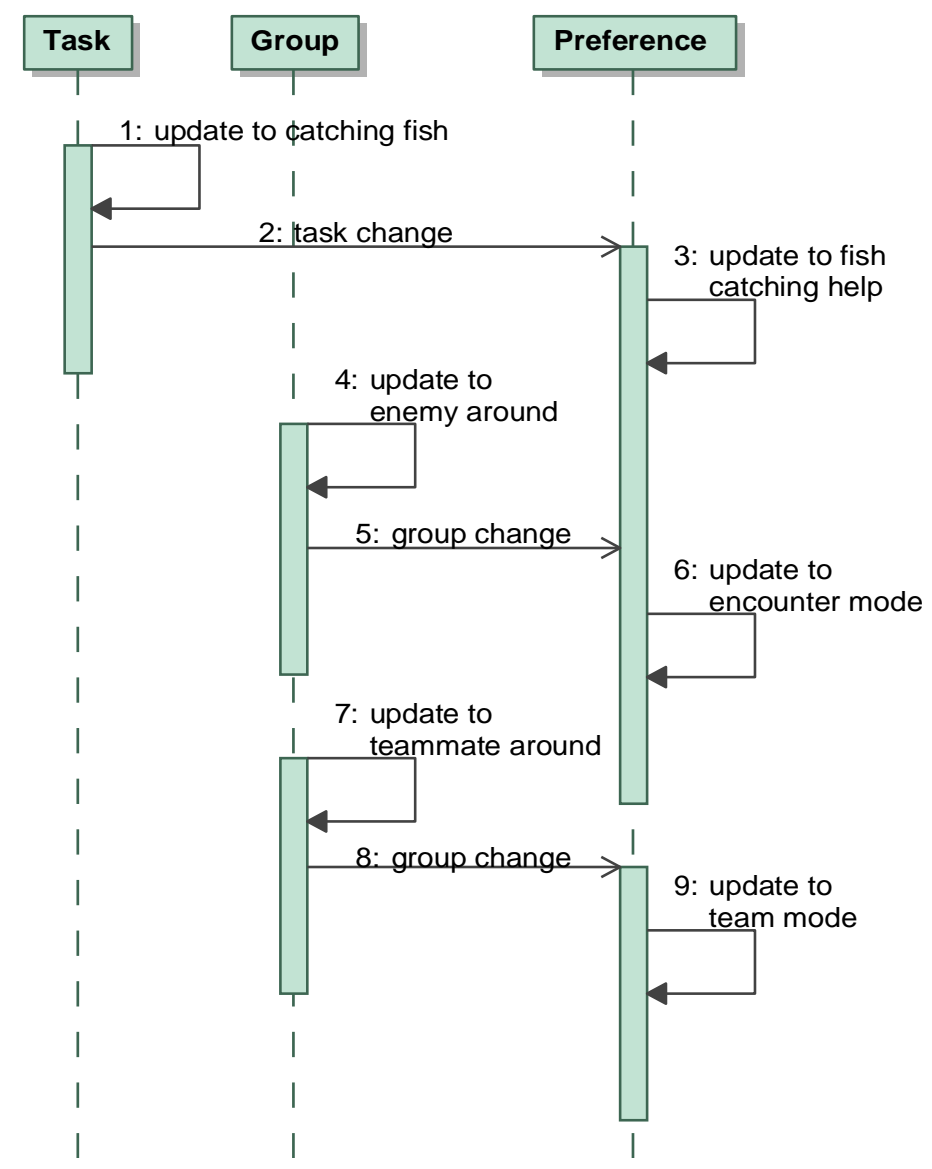

Figure 27 Sequence diagram describing dynamic interactions between tasks, groups and preferences in the virtual gaming environment in the mobile augmented reality gaming scenario

\subsection{Conclusion}

This chapter has presented four case studies which illustrated the usefulness and applicability of the model. Each case study instantiated part of the context model to its specific application domain, and the results were shown through object and sequence diagrams. The first case study on mobile ehealthcare illustrated role adaptations triggered by tasks and environments. The changes propagated to the preference component which updated itself to facilitate the new role. The second case study on mobile commerce illustrated the preference adaptations triggered by roles, tasks, and groups. The third case study on mobile tourism focused on the impact of social relationships and groups on user preferences. The group of users that are relevant to the situation may be ones that are currently around such as other tourists or ones that are away but are in the user's social networks. The fourth case 
study on mobile augmented reality gaming illustrated the dynamic interactions among contextual elements in the virtual gaming environment. These case studies together illustrated all of the dynamic interactions captured by the context model and also showcased the potential new types of mobile context-aware applications that can be enabled by the context model proposed. 


\section{Chapter 6}

\section{Conclusion and Future Work}

\subsection{Conclusion}

This thesis has presented the following contributions:

- A survey of existing context-aware systems;

- A design of a dynamic user-centric context model; and

- Four case studies in the mobile application domain.

A survey of existing context-aware systems was conducted based on the fundamental elements in context models and the functionality provided by context-aware systems. The survey contributes in a fundamental element and a functionality classification frameworks. The context-aware system survey presents the state-of-the-art in context aware systems, and the results of the survey identify gaps in the existing works in context-aware systems. There is a need for design abstractions that describe dynamic interactions between user context elements as well as virtual environments.

Further, this thesis has proposed a dynamic user-centric context model which is applied to the mobile application domain. The context model considers the dynamic interactions between contextual elements, and thus encapsulates dynamic behaviours of context-aware systems. It models essential user context abstractions such as roles, preferences, and social relationships as well as the possibility of dynamic interactions involving virtual environments. The proposed design can be used to develop mobile context-aware systems that are highly dynamic and user-centric.

Finally, this thesis has presented four case studies in the domain of mobile e-healthcare, mobile commerce, mobile tourism, and mobile augmented reality gaming. These case studies illustrate the dynamic interactions between contextual elements as described in the context model. They show the 
applicability and usage of the model as well as the potential context-aware mobile applications that can be instantiated from the context model.

\subsection{Future Work}

In the future, first, we would like to extend 5W1H (who, what, where, when, how, and why) [40] and the survey of context-aware systems to refine this work further. We may create a mapping from our proposed fundamental classification framework to the classification proposed by $5 \mathrm{~W} 1 \mathrm{H}$. We may also extend $5 \mathrm{~W} 1 \mathrm{H}$ to support dynamic interactions involving user roles and investigate further the functionality that can be included by modelling dynamic user-centric contexts.

Second, we would like to incorporate different perspectives of context-aware systems by clustering the contextual elements in our context model in a more general way. We would like to start with an event view of the model. Event-based interactions provide loose coupling between contextual elements and can easily support dynamic interactions between contextual elements. The event view makes the events and communications within the model explicit and it can include contextual elements such as calendar, time, and tasks. To extend further the model, we may include a service view which deals with applications and services as well as a system view which deals with environments, devices, and networks. Our context model and case studies are currently modelled through Unified Modeling Language, and we may deploy the interaction-based modelling techniques to represent the interactions between user context elements [65].

Third, we would like to extend our work to protect user privacy and ensure the security of user data as well as consider the quality of context data. Another future research direction is to explore the potential of applying our context model to learn and predict user behaviours.

Fourth, we may deploy context evaluation metrics. For example, we may evaluate our context model based on criteria such as the extensibility and scalability of the model. The essential properties of the context model could be verified through a model verification process to ensure that the model would function as expected. By evaluating the context model and verifying its essential properties, we can illustrate further the applicability and usefulness of our model.

Finally, we would also like to extend the case studies to study the contextual elements in our context model by applying the model to different domains. A prototype tool could support the representation and execution of the model. Based on the prototype, we could conduct user studies to 
understand user needs and enable potential new functions. Distributed event-based systems provide a way to integrate the existing context-aware systems into highly dynamic environments, and we may implement the prototype in terms of these event-based systems. 


\section{References}

[1] A. K. Dey and G. D. Abowd, "Towards a better understanding of context and context-awareness," in Proceedings of CHI 2000 Workshop on the what, Who, Where, and how of Context-Awareness, The Hague, The Netherlands, 2000.

[2] S. Baek, H. Lee, S. Lim and J. Huh, "Managing mechanism for service compatibility and interaction issues in context-aware ubiquitous home," IEEE Transactions on Consumer Electronics, vol. 51, pp. 524-528, 2005.

[3] M. Danninger and R. Stiefelhagen, "A context-aware virtual secretary in a smart office environment," in Proceeding of the 16th ACM International Conference on Multimedia, Vancouver, British Columbia, Canada, 2008, pp. 529-538.

[4] D. Petrelli and E. Not, "User-centred design of flexible hypermedia for a mobile guide: Reflections on the HyperAudio experience," User Modeling and User-Adapted Interaction, vol. 15, pp. 303-338, 2005.

[5] T. Strang, C. Linnhoff-Popien and K. Frank, "Applications of a context ontology language," in Proceedings of International Conference on Software, Telecommunications and Computer Networks, Split/Croatia, Venice/Italy, Ancona/Italy, Dubrovnik/Croatia, 2003, pp. 14-18.

[6] J. Cano, P. Manzoni and C. -. Toh, "UbiqMuseum: A Bluetooth and Java based context-aware system for ubiquitous computing," Wireless Personal Communications, vol. 38, pp. 187-202, 2006.

[7] S. Kurkovsky, V. Zanev and A. Kurkovsky, "SMMART, a context-aware mobile marketing application: Experiences and lessons," Embedded and Ubiquitous Computing, vol. 3823/2005, pp. 141-150, 2005.

[8] J. E. Bardram and N. Nørskov, "A context-aware patient safety system for the operating room," in Proceedings of the 10th International Conference on Ubiquitous Computing, Seoul, Korea, 2008, pp. 272-281.

[9] N. Fröhlich, A. Meier, T. Möller, M. Savini, H. Schuldt and J. Vogt, "LoCa - Towards a contextaware infrastructure for eHealth applications," in Proceedings of 15th International Conference on Distributed Multimedia Systems, Redwood City, San Francisco Bay, USA, 2009, pp. 52-63.

[10] A. K. Dey, G. D. Abowd and D. Salber, "A conceptual framework and a toolkit for supporting the rapid prototyping of context-aware applications," International Journal of Human-Computer Interaction, vol. 16, pp. 97-166, 2001.

[11] D. Salber, A. K. Dey and G. D. Abowd, "The context toolkit: Aiding the development of context-enabled applications," in Proceedings of the SIGCHI Conference on Human Factors in Computing Systems, Pittsburgh, Pennsylvania, United States, 1999, pp. 434-441. 
[12] M. Vuković, E. Kotsovinos and P. Robinson, "An architecture for rapid, on-demand service composition," Service Oriented Computing and Applications, vol. 1, pp. 197-212, 12/30, 2007.

[13] M. R. Blaha and J. R. Rumbaugh, Object-Oriented Modeling and Design with UML. Upper Saddle River, New Jersey: Prentice Hall, 2004.

[14] R. T. Azuma, "A survey of augmented reality," Presence: Teleoperators and Virtual Environments, vol. 6, pp. 355-385, 1997.

[15] P. Honkamaa, J. Jäppinen and C. Woodward, "A lightweight approach for augmented reality on camera phones using 2D images to simulate 3D," in Proceedings of the 6th International Conference on Mobile and Ubiquitous Multimedia, Oulu, Finland, 2007, pp. 155-159.

[16] K. Al-Mekhlafi, X. Hu and Z. Zheng, "An approach to context-aware mobile Chinese language learning for foreign students," in Proceedings of the 8th International Conference on Mobile Business, Dalian, China, 2009, pp. 340-346.

[17] H. Ogata and Y. Yano, "Context-aware support for computer-supported ubiquitous learning," in Proceedings of the 2nd IEEE International Workshop on Wireless and Mobile Technologies in Education, Taoyuan, Taiwan, 2004, pp. 27-34.

[18] L. Ramaswamy, D. P., R. Polavarapu, K. Gunasekera, D. Garg, K. Visweswariah and S. Kalyanaraman, "CAESAR: A context-aware, social recommender system for low-end mobile devices," in Proceedings of the 2009 10th International Conference on Mobile Data Management: Systems, Services and Middleware, Taipei, Taiwan, 2009, pp. 338-347.

[19] Y. Sumi, T. Etani, S. Fels, N. Simonet, K. Kobayashi and K. Mase, "C-MAP: Building a contextaware mobile assistant for exhibition tours," in Community Computing and Support Systems, Social Interaction in Networked Communities [the Book is Based on the Kyoto Meeting on Social Interaction and Communityware, Held in Kyoto, Japan, in June 1998], 1998, pp. 137-154.

[20] K. Cheverst, N. Davies, K. Mitchell and A. Friday, "Experiences of developing and deploying a context-aware tourist guide: The GUIDE project," in Proceedings of the 6th Annual International Conference on Mobile Computing and Networking, Boston, Massachusetts, United States, 2000, pp. 20-31.

[21] G. D. Abowd, C. G. Atkeson, J. Hong, S. Long, R. Kooper and M. Pinkerton, "Cyberguide: A mobile context-aware tour guide," Wireless Networks, vol. 3, pp. 421-433, 1997.

[22] G. Reitmayr and D. Schmalstieg, "Collaborative augmented reality for outdoor navigation and information browsing," in In Proceedings of the Symposium on Location Based Services and TeleCartography, Vienna, Austria, 2004, pp. 31-41.

[23] O. Rashid, I. Mullins, P. Coulton and R. Edwards, "Extending cyberspace: Location based games using cellular phones," Computers in Entertainment, vol. 4, pp. 3-20, 2006. 
[24] P. Coppola, V. D. Mea, L. D. Gaspero, D. Menegon, D. Mischis, S. Mizzaro, I. Scagnetto and L. Vassena, "The context-aware browser," IEEE Intelligent Systems, vol. 25, pp. 38-47, 2010.

[25] H. Chen, T. Finin and A. Joshi, "Using OWL in a pervasive computing broker," in Proceedings of Workshop on Ontologies in Agent Systems, Melbourne, Victoria, Australia, 2003, pp. 9-16.

[26] S. J. H. Yang, A. P. M. Huang, R. Chen, S. S. Tseng and Y. Shen, "Context model and context acquisition for ubiquitous content access in U-learning environments," in Proceedings of the IEEE International Conference on Sensor Networks, Ubiquitous, and Trustworthy Computing, 2006, pp. 78-83.

[27] M. A. Strimpakou, I. G. Roussaki and M. E. Anagnostou, "A context ontology for pervasive service provision," in Proceedings of the 20th International Conference on Advanced Information Networking and Applications, Vienna, Austria, 2006, pp. 775-779.

[28] B. Schilit, N. Adams and R. Want, "Context-aware computing applications," in Proceedings of the 1st Workshop on Mobile Computing Systems and Applications, 1994, pp. 85-90.

[29] K. Cheverst, K. Mitchell and N. Davies, "Design of an object model for a context sensitive tourist GUIDE," Computers and Graphics, vol. 23, pp. 883-891, 12, 1999.

[30] K. Henricksen, J. Indulska and A. Rakotonirainy, "Modeling context information in pervasive computing system," in Proceedings of the 1st International Conference on Pervasive Computing, 2002, pp. 167-180.

[31] H. Chen, F. Perich, T. Finin and A. Joshi, "SOUPA: Standard ontology for ubiquitous and pervasive applications," in Proceedings of the 1st Annual International Conference on Mobile and Ubiquitous Systems: Networking and Services, Boston, MA, 2004, pp. 258-267.

[32] N. Savio and J. Braiterman, "Design sketch: The context of mobile interaction," in Proceedings of International Journal of Mobile Marketing, Singapore, 2007, pp. 5-7.

[33] P. Korpipää and J. Mantyjarvi, "An ontology for mobile device sensor-based context awareness," in Proceedings of the 4th International and Interdisciplinary Conference on Modeling and using Context, Stanford, CA, USA, 2003, pp. 451-458.

[34] M. A. Muñoz, M. Rodríguez, J. Favela, A. I. Martinez-Garcia and V. M. González, "Contextaware mobile communication in hospitals," Computer, vol. 36, pp. 38-46, 2003.

[35] A. Echtibi, M. J. Zemerly and J. Berri, "Murshid: A mobile tourist companion," in Proceedings of the 1st International Workshop on Context-Aware Middleware and Service, Dublin, Ireland, 2009, pp. 6-11.

[36] T. Gu, H. K. Pung and D. Q. Zhang, "A service-oriented middleware for building context-aware services," Journal of Network and Computer Applications, vol. 28, pp. 1-18, 2005. 
[37] Z. Hwang, Y. Uhm, M. Lee, Y. Kim, G. Kim and S. Park, "A context model by ontology and rule for offering the user-centric services in ubiquitous computing," in Proceedings of the 2nd International Conference on Convergence Information Technology, Gyeongju, Republic of Korea, 2007, pp. 77-82.

[38] D. Preuveneers, d. B. Van, D. Wagelaar, A. Georges, P. Rigole, T. Clerckx, Y. Berbers, K. Coninx, V. Jonckers and K. De Bosschere, "Towards an extensible context ontology for ambient intelligence," in Proceedings of the 2nd European Symposium on Ambient Intelligence, Eindhoven, The Netherlands, 2004, pp. 148-159.

[39] S. Jang and W. Woo, "5W1H: Unified user-centric context," in Proceedings of the 7th International Conference on Ubiquitous Computing, Tokyo, Japan, 2005.

[40] D. Hong, H. R. Schmidtke and W. Woo, "Linking context modelling and contextual reasoning," in Proceedings of the 4th International Workshop on Modeling and Reasoning in Context, Roskilde, Denmark, 2007, pp. 37-48.

[41] S. Jang and W. Woo, "Ubi-UCAM: A unified context-a ware application model," in Proceedings of the 4th International and Interdisciplinary Conference on Modeling and using Context, Stanford, CA, USA, 2003, pp. 178-189.

[42] A. Tafat, M. Courant and B. Hirsbrunner, "A generic coordination model for pervasive computing based on semantic Web languages," in Proceedings of the 9th International Conference on Applications of Natural Language to Information System, Salford, UK, 2004, pp. 265-275.

[43] Y. Chang, P. Alencar, E. Barrenechea, R. Blanco and D. Cowan, "Context-aware systems: Models and functionality," in Handbook of Research on Mobile Software Engineering: Design Implementation and Emergent Applications, P. Alencar and D. Cowan, Eds. Engineering Science Reference, 2010.

[44] J. Hong, E. Suh and S. Kim, "Context-aware systems: A literature review and classification," Expert Systems with Applications, vol. 36, pp. 8509-8522, 5, 2009.

[45] R. Krummenacher and T. Strang, "Ontology-based context modeling," in Proceedings of the 3rd Workshop on Context Awareness for Proactive Systems, Guildford, United Kingdom, 2007.

[46] M. Baldauf, S. Dustdar and F. Rosenberg, "A survey on context-aware systems," International Journal of Ad Hoc and Ubiquitous Computing, vol. 2, pp. 263-277, 2007.

[47] C. Bolchini, C. A. Curino, E. Quintarelli, F. A. Schreiber and L. Tanca, "A data-oriented survey of context models," Special Interest Group on Management of Data, vol. 36, pp. 19-26, 2007.

[48] J. Ye, L. Coyle, S. Dobson and P. Nixon, "Ontology-based models in pervasive computing systems," The Knowledge Engineering Review, vol. 22, pp. 315-347, 2007. 
[49] T. Gu, X. H. Wang, H. K. Pung and D. Q. Zhang, "An ontology-based context model in intelligent environments," in Proceedings of Communication Networks and Distributed Systems Modeling and Simulation Conference, San Diego, California, USA, 2004, pp. 270-275.

[50] I. Roussaki, M. Strimpakou, C. Pils, N. Kalatzis and M. Anagnostou, "Hybrid context modeling: A location-based scheme using ontologies," in Proceedings of the 4th Annual IEEE International Conference on Pervasive Computing and Communications Workshops, Pisa, Italy, 2006, pp. 2-7.

[51] J. Coutaz, J. L. Crowley, S. Dobson and D. Garlan, "Context is key," Communications of the ACM, vol. 48, pp. 49-53, 2005.

[52] P. Korpipää, J. Mäntyjärvi, J. Kela, H. Keränen and E. Malm, "Managing context information in mobile devices," IEEE Pervasive Computing, vol. 2, pp. 42-51, 2003.

[53] A. Held, S. Buchholz and A. Schill, "Modeling of context information for pervasive computing applications," in Proceedings of the 6th World Multiconference on Systemics, Cybernetics and Informatics, Orlando, USA, 2002.

[54] X. H. Wang, D. Q. Zhang, T. Gu and H. K. Pung, "Ontology based context modeling and reasoning using OWL," in Proceedings of the 2nd IEEE Annual Conference on Pervasive Computing and Communications Workshops, Orlando, Florida, USA, 2004, pp. 18-22.

[55] T. Strang, C. Linnhoff-Popien and K. Frank, "CoOL: A context ontology language to enable contextual interoperability," in Distributed Applications and Interoperable Systems Anonymous London, UK: Springer Berlin / Heidelberg, 2003, pp. 236-247.

[56] J. Coutaz, A. Dearle, S. Dupuy-Chessa, G. N. C. Kirby, C. Lachenal, R. Morrison, G. Rey and E. Zirintsis, "Working document on gloss ontology," Technical Report D9.2, Global Smart Spaces Project IST-2000-26070, 2003.

[57] A. Dearle, G. Kirby, R. Morrison, A. McCarthy, K. Mullen, Y. Yang, R. Connor, P. Welen and A. Wilson, "Architectural support for global smart spaces," in Proceedings of the 4th International Conference on Mobile Data Management, Melbourne, Australia, 2003, pp. 153-164.

[58] B. Pernici, "MAIS: Multichannel adaptive information systems," in Proceedings of the 4th International Conference on Web Information Systems Engineering, Roma, Italy, 2003, pp. 303-305.

[59] D. Petrelli, E. Not, C. Strapparava, O. Stock and M. Zancanaro, "Modeling context is like taking pictures," in Proceedings of CHI 2000 Workshop on the what, Who, Where, and how of ContextAwareness, The Hague, The Netherlands, 2000.

[60] D. Preuveneers and Y. Berbers, "Adaptive context management using a component-based approach," in Proceedings of the 5th International Conference on Distributed Applications and Interoperable Systems, Athens, Greece, 2005, pp. 14-26. 
[61] I. Millard, D. De Roure and N. Shadbolt, "The use of ontologies in contextually aware environments," in Proceedings of the 1st International Workshop on Advanced Context Modelling, Reasoning and Management, Nottingham, UK, 2004, pp. 42-47.

[62] J. L. Crowley, J. Coutaz, G. Rey and P. Reignier, "Perceptual components for context aware computing," in Proceedings of the 4th International Conference on Ubiquitous Computing, Göteborg, Sweden, 2002, pp. 117-134.

[63] E. Christopoulou and A. Kameas, "GAS ontology: An ontology for collaboration among ubiquitous computing devices," International Journal of Human-Computer Studies, vol. 62, pp. 664685, 2005.

[64] Y. Chang, E. Barrenechea and P. and Alencar, "Dynamic user-centric mobile context model," in Proceedings of the 4th International Workshop on Context Modeling and Management for Smart Environments, Thunder Bay, Canada, 2010.

[65] D. Cram, B. Fuchs, Y. Prié and A. Mille, "An approach to user-centric context-aware assistance based on interaction traces," in Proceedings of the 5th International Workshop on Modelling and Reasoning in Context, Delft, The Netherlands, 2008, pp. 89-101. 